\title{
VALUATION OF HYBRID FINANCIAL AND ACTUARIAL PRODUCTS IN LIFE INSURANCE BY A NOVEL THREE-STEP METHOD
}

\author{
BY \\ Griselda Deelstra, Pierre Devolder, Kossi Gnameho And \\ PETER Hieber (iD
}

\begin{abstract}
Financial products are priced using risk-neutral expectations justified by hedging portfolios that (as accurate as possible) match the product's payoff. In insurance, premium calculations are based on a real-world best-estimate value plus a risk premium. The insurance risk premium is typically reduced by pooling of (in the best case) independent contracts. As hybrid life insurance contracts depend on both financial and insurance risks, their valuation requires a hybrid valuation principle that combines the two concepts of financial and actuarial valuation. The aim of this paper is to present a novel three-step projection algorithm to valuate hybrid contracts by decomposing their payoff in three parts: a financial, hedgeable part, a diversifiable actuarial part, and a residual part that is neither hedgeable nor diversifiable. The first two parts of the resulting premium are directly linked to their corresponding hedging and diversification strategies, respectively. The method allows for a separate treatment of unsystematic, diversifiable mortality risk and systematic, aggregate mortality risk related to, for example, epidemics or population-wide improvements in life expectancy. We illustrate our method in the case of CAT bonds and a pure endowment insurance contract with profit and compare the three-step method to alternative valuation operators suggested in the literature.
\end{abstract}

\section{KEYWORDS}

Financial risk, actuarial valuation, contract valuation, incomplete markets, risk decomposition, hedging.

\section{INTRODUCTION}

The valuation of future cash flows is a fundamental problem in both finance and actuarial science. In a classical deterministic setting, the problem is trivial: the value is then simply given by the discounted future cash flows (see, e.g.,

Astin Bulletin 50(3), 709-742. doi:10.1017/asb.2020.25 (C) 2020 by Astin Bulletin. All rights reserved. This is an Open Access article, distributed under the terms of the Creative Commons Attribution licence (http://creativecommons.org/licenses/by/4.0/), which permits unrestricted re-use, distribution, and reproduction in any medium, provided the original work is properly cited. 
Boyle and Schwartz, 1977; Brennan and Schwartz, 1979; Gerber, 1997). However, as soon as the cash flows become uncertain, different methodologies are necessary. In particular, two different philosophies of pricing are present in finance and in insurance, respectively. The classical paradigm of pricing in insurance is based on the law of large numbers: the basic premium is equal to the discounted expectation of the risk under the real probability measure plus a safety margin (for instance, the standard deviation principle (see, e.g., Kaas et al., 2008; Laeven and Goovaerts, 2008; Rotar, 2014) or the Solvency II cost of capital (see, e.g., Keller and Luder, 2004)). This valuation, based on a "mean" logic, comes directly from the underlying strategy of the insurer to limit its own risk, namely the mutualization of a big number of diversifiable independent risks in an insurance pool. The right strategy for the insurer, in line with this pricing tool, is then to increase the size of the pool. In finance, the approach is quite different; pricing in complete markets is based on discounted expectation of future payoffs but now under an equivalent martingale measure (for instance, the risk-neutral measure). Also here, the valuation methodology can be justified by an underlying strategy: the well-known hedging technique. The basic strategy is then to use replicating portfolios whose price corresponds to the risk-neutral expectation of the discounted payoff. In practice, there are many reasons why this dichotomy of philosophy between finance and insurance is quite challenging. First, the main assumptions behind the two paradigms can be discussed: in finance, markets are very often incomplete and a perfect hedge remains very difficult or even impossible to achieve. In insurance, risks are not always perfectly diversifiable (take catastrophic (CAT) risks or longevity risks as an example) violating the assumptions of the law of large numbers. Secondly, hybrid products based on a combination of actuarial and financial components are more and more present on the markets (for instance, life insurance products with optional payoffs) and require then a new adjusted valuation principle. Different principles have been proposed in the literature in order to price these hybrid products (see, e.g., Møller, 2002; Malamud et al., 2008; Möhr, 2011; Pelsser and Stadje, 2014; Pelsser and Ghalehjooghi, 2016; Dhaene et al., 2017; Barigou and Dhaene, 2019; Delong et al., 2019a,b; Engsner et al., 2020 and many others). Our article contributes to this literature by proposing a novel decomposition method with a clear separation of risks that are hedgeable on financial markets, risks that can be reduced by pooling, and a remaining part evaluated by taking into account solvency guidelines. This method is based upon hedging and diversification strategies, respectively, for the first two parts. In particular, it allows to distinguish between the different types of mortality risks (unsystematic, diversifiable mortality risk and aggregate, systematic mortality risk, see also Chen et al., 2019). In this article, we focus, in a static 1-period framework, upon a general evaluation method to cope with hybrid life products, while working in a complete financial market and supposing independence between financial and actuarial risks. Our method is based on a three-step projection algorithm, consistent with the concepts of risk-neutral pricing in finance as well as with the pooling principle 
in insurance, and coherent with the classical financial and actuarial valuation. Moreover, we can produce a coherent strategy to manage the risk. The basic idea of the method is to decompose any future stochastic cash flow into three additive components:

- part 1: a financial, hedgeable part that can be priced under an equivalent martingale measure following the finance paradigm. This part can be replicated by existing assets and contains no risk (when properly hedged), neither systematic nor unsystematic.

- part 2: a diversifiable part that can be priced under the real probability measure following insurance principles. This part contains only diversifiable risks that can be asymptotically canceled by increasing the size of the pool of risks.

- part 3: a part which is neither hedgeable nor diversifiable. This part can be based on insurance risk and financial risks and requires an adapted (hybrid) methodology.

We provide a comparison to other hybrid valuation operators suggested in the literature (e.g., Møller, 2002; Pelsser and Stadje, 2014) and discuss their implications. Among others, we want to stress that hybrid life products do usually not allow to fully disentangle financial and actuarial risks. Thus, even in the case of a complete financial market, it is not possible to fully eliminate financial risks.

We organize the remainder of this article as follows: in Section 2, we introduce the general model framework, the payoff of hybrid life products and the basic concept of a financial and actuarial valuation principle. In Section 3, we show how the product's payoff can be decomposed in a financial, an actuarial and a residual part. This decomposition is used in Section 4 to introduce the three-step method. We introduce other approaches suggested in the literature and provide a comparison and axiomatic assessment of these valuation operators. In Sections 5 and 6, we apply the different valuation operators to valuate general life insurances and securitization as, for example, CAT bonds. In Section 7, we include a numerical illustration with discussion of the different evaluation operators, and this in the setting of a pure endowment life insurance contract with profit. Section 8 discusses the stability of our results if certain simplifying assumptions are relaxed. Section 9 concludes.

\section{Model SETUP AND ASSUMPTIONS}

Throughout, we operate on a finite time interval $[0, T]$. We consider a pool of $n \in \mathbb{N}$ insured individuals with remaining lifetimes $\tau:=\left(\tau_{1}, \tau_{2}, \ldots, \tau_{n}\right)$. Mortality risk consists of two parts: systematic or aggregate mortality risk referring to risk factors that lead to a population-wide in- or decrease in life expectancy and unsystematic mortality risk taking into account random deviations from the population-wide mortality experience. On the probability 
space $(\Omega, \mathcal{F}, \mathbb{P})$, we consider a filtration $\left(\mathcal{G}_{t}\right)_{t}$ that is generated by the systematic mortality risk factors. For each individual $j=1,2, \ldots, n$, we consider $\mathcal{I}_{t}^{j}:=\sigma\left(\mathbb{1}_{\left\{\tau_{j} \leq u\right\}}: u \leq t\right)$. Under the real-world measure $\mathbb{P}$, the $\sigma$-fields $\mathcal{I}^{j}$ (for $j=1,2, \ldots, n)$ are assumed to be conditionally independent given the systematic mortality information contained in $\mathcal{G}_{T}$, that is, for any $T>0$ and $t \in[0, T]$, we have that

$$
\mathbb{P}\left(\tau_{1}>t, \tau_{2}>t, \ldots, \tau_{n}>t \mid \mathcal{G}_{T}\right)=\prod_{j=1}^{n} \mathbb{P}\left(\tau_{j}>t \mid \mathcal{G}_{T}\right) .
$$

This model framework was originally proposed in credit risk, see, for example, Bielecki and Rutkowski (2004), p. 268ff. The enlarged time- $t$ actuarial filtration is then defined as $\mathcal{F}_{t}^{a}:=\mathcal{G}_{t} \vee\left(\vee_{j=1}^{n} \mathcal{I}_{t}^{j}\right)$. We further denote the time- $t$ filtration of financial risk by $\mathcal{F}_{t}^{f}$, defined on $\Omega$. From this, the full information filtration is $\mathcal{F}_{t}:=\mathcal{F}_{t}^{a} \vee \mathcal{F}_{t}^{f}$ for all $t \in[0, T]$. The number of policyholders at any time $t \in[0, T]$ is denoted by $N_{t}:=\sum_{j=1}^{n} \mathbb{1}_{\left\{\tau_{j} \geq t\right\}}$. Under the real-world measure $\mathbb{P}$, we assume that financial and actuarial risks are independent. On the product space $\left(\Omega=\Omega^{a} \times \Omega^{f}, \mathcal{F}\right)$, we choose the product measure $\mathbb{P}:=\mathbb{P}^{a} \times \mathbb{P}^{f}$, where throughout this article - the superscripts ${ }^{a}$ and ${ }^{f}$ represent quantities related to actuarial and financial risks, respectively.

Hybrid life products. Let us introduce functions $g_{j}(\tau):(0, \infty) \rightarrow \mathbb{R}^{+}$and $h_{j}(s)$ : $(0, \infty) \rightarrow \mathbb{R}^{+}, j=1,2, \ldots, n$. We consider hybrid life products that can be represented by the time- $T$ payoffs

$$
H_{T}^{n}=\frac{1}{n} \sum_{j=1}^{n} g_{j}\left(\tau_{j}\right) \cdot h_{j}\left(S_{T}\right),
$$

where $S_{T}$ denotes the time- $T$ value of a financial asset, $h_{j}\left(S_{T}\right)$ is $\mathcal{F}_{T}^{f}$-measurable, and $g_{j}\left(\tau_{j}\right)$ is $\mathcal{F}_{T}^{a}$-measurable. We discuss generalizations of the payoff (2.1) in Section 6.

We denote the set of contingent claims (2.1) by $\mathcal{H}_{T}$. The subclass of financial claims (obtained by setting $g_{j}\left(\tau_{j}\right)=1$ for $j=1,2, \ldots, n$ in (2.1)) is denoted by $\mathcal{H}_{T}^{f}$, while the subclass of actuarial claims (obtained by setting $h_{j}\left(S_{T}\right)=1$ for $j=1,2, \ldots, n$ in (2.1)) is referred to as $\mathcal{H}_{T}^{a}$.

Product valuation. We consider a complete financial market containing risky assets and a risk-free bank account with constant risk-free rate $r$. Further, we assume that there exists an equivalent martingale measure $\mathbb{Q}^{f} \sim \mathbb{P}^{f}$ such that $\mathrm{d} \mathbb{Q}^{f} / \mathrm{d} \mathbb{P}^{f} \in \mathcal{H}_{T}^{f}$. The value of a financial claim $H_{T}^{n} \in \mathcal{H}_{T}^{f}$ is then given by its riskneutral expectation, that is

$$
\pi_{0}^{f}\left(H_{T}^{n} \mid \mathcal{F}_{0}\right):=\mathbb{E}_{\mathcal{F}_{0}}^{\mathbb{Q}_{0}}\left[e^{-r T} H_{T}^{n}\right],
$$


where we denote conditional expectations by $\mathbb{E}_{\mathcal{F}_{\mathcal{F}}}^{\mathbb{Q}_{f}}:=\mathbb{E}^{\mathbb{Q}^{f}}\left[\cdot \mid \mathcal{F}_{t}\right]$. We extend (2.2) to define a set of financial valuation operators for a general claim $H_{T}^{n} \in \mathcal{H}_{T}$ as ${ }^{1}$

$$
\pi_{0}^{f}\left(H_{T}^{n} \mid \mathcal{F}_{0}\right):=\mathbb{E}_{\mathcal{F}_{0}}^{\mathbb{P}^{a} \times \mathbb{Q}^{f}}\left[e^{-r T} H_{T}^{n}\right] .
$$

We further consider a set of actuarial valuation operators. Using the standard deviation principle, the value of a purely actuarial claim $H_{T}^{n} \in \mathcal{H}_{T}^{a}$ is

$$
\pi_{0}^{a}\left(H_{T}^{n} \mid \mathcal{F}_{0}\right):=e^{-r T}\left(\mathbb{E}_{\mathcal{F}_{0}}^{\mathbb{P}^{a}}\left[H_{T}^{n}\right]+\frac{\alpha}{2} \sqrt{\operatorname{Var}_{\mathcal{F}_{0}}^{\mathbb{P} a}\left(H_{T}^{n}\right)}\right),
$$

where $\alpha>0$ is the risk aversion coefficient. Again, we extend this to general claims $H_{T}^{n} \in \mathcal{H}_{T}$ and define under the real-world measure $\mathbb{P}:=\mathbb{P}^{a} \times \mathbb{P}^{f}$ that

$$
\pi_{0}^{a}\left(H_{T}^{n} \mid \mathcal{F}_{0}\right):=e^{-r T}\left(\mathbb{E}_{\mathcal{F}_{0}}^{\mathbb{P}^{a} \times \mathbb{P}^{f}}\left[H_{T}^{n}\right]+\frac{\alpha}{2} \sqrt{\operatorname{Var}_{\mathcal{F}_{0}}^{\mathbb{P}^{a} \times \mathbb{P}^{f}}\left(H_{T}^{n}\right)}\right) .
$$

Formulas (2.3) and (2.5) could be applied to any hybrid product but they are clearly contradictory. The purpose of this article is to propose a general evaluation method for any hybrid claim, consistent with formula (2.2) for pure financial products $H_{T}^{n} \in \mathcal{H}_{T}^{f}$, consistent with formula (2.4) for pure diversifiable actuarial claims $H_{T}^{n} \in \mathcal{H}_{T}^{a}$ and able to valuate claims neither completely hedgeable nor diversifiable. We want also to develop a product valuation reflecting strategies to treat the underlying risk.

The purpose of this paper is to propose an evaluation method for the hybrid claim $H_{T}^{n}$ defined in (2.1), which takes into account strategies to treat the underlying risks based on ideas from finance and insurance.

For a purely financial, hedgeable contract, it is well known that one should apply a purely financial premium that does not include any additional risk margins. This property (market consistency) takes into account that payoffs that can be liquidly replicated on financial markets should not carry any risk as they can be liquidated at any time. Similarly, purely actuarial pools of independent risks must be valued by purely actuarial valuation principles. Here, risks cannot be eliminated on financial markets, and the valuation operator has to add appropriate risk margins for this unhedgeable but diversifiable risk (a property we call actuarial valuation operator). Definition 2.1 introduces both concepts in a formal way.

Definition 2.1 (Market-consistent and actuarial valuation operator).

- The valuation operator is market-consistent if, for any financial, hedgeable claim $h_{1}\left(S_{T}\right) \in \mathcal{H}_{T}^{f}$ and any claim $H_{T}^{n} \in \mathcal{H}_{T}$, it holds that

$$
\pi_{0}\left(H_{T}^{n}+h_{1}\left(S_{T}\right)\right)=\pi_{0}\left(H_{T}^{n}\right)+\pi_{0}^{f}\left(h_{1}\left(S_{T}\right) \mid \mathcal{F}_{0}\right) .
$$


- The valuation operator is actuarial if, for an insurance portfolio whose claims are independent (i.e., a portfolio of insureds whose remaining lifetimes $\tau_{j}$, $j=1,2, \ldots, n$, are independent and identically distributed (i.i.d.)) and an actuarial claim $g_{T}^{n}:=\frac{1}{n} \sum_{j=1}^{n} g_{j}\left(\tau_{j}\right) \in \mathcal{H}_{T}^{a}$, the valuation operator is given by an actuarial valuation operator, that is,

$$
\pi_{0}\left(g_{T}^{n}\right)=\pi_{0}^{a}\left(g_{T}^{n} \mid \mathcal{F}_{0}\right) .
$$

It is desirable that any valuation operator is market-consistent and actuarial (see, e.g., Delong et al., 2019a,b for a detailed discussion).

\section{PAYOFF DECOMPOSITION OF HYBRID CLAIMS}

In this article, we discuss on how to choose a valuation operator for hybrid products $H_{T}^{n} \in \mathcal{H}_{T}$ that contain appropriate risk margins for the product's various risks. We want to suggest a valuation operator that decomposes the contract's payoff in hedgeable financial risk, diversifiable actuarial risk, and a third component that relates to unhedgeable and undiversifiable risk, for example, due to aggregate mortality risk. Therefore, as a first step, we decompose the mortality-risk-related part of the payoff as

$$
\begin{aligned}
g_{j}\left(\tau_{j}\right)= & \underbrace{\mathbb{E}_{\mathcal{F}_{0} \times \mathbb{P}^{f}}^{\mathbb{P}^{a}}\left[g_{j}\left(\tau_{j}\right)\right]}_{\text {best-estimate }}+\underbrace{\left(g_{j}\left(\tau_{j}\right)-\mathbb{E}_{\mathcal{F}_{0} \vee \mathcal{G}_{T}}^{\mathbb{P}^{a} \times \mathbb{P}^{f}}\left[g_{j}\left(\tau_{j}\right)\right]\right)}_{\text {unsystematic deviation }} \\
& +\underbrace{\left(\mathbb{E}_{\mathcal{F}_{0} \vee \mathcal{G}_{T}}^{\mathbb{P}^{a} \times \mathbb{P}^{f}}\left[g_{j}\left(\tau_{j}\right)\right]-\mathbb{E}_{\mathcal{F}_{0}}^{\mathbb{P}^{a} \times \mathbb{P}^{f}}\left[g_{j}\left(\tau_{j}\right)\right]\right)}_{\text {systematic deviation }} .
\end{aligned}
$$

The suggested decomposition depends on the underlying model for (un)systematic mortality risk. In Section 5 and the numerical part of this article, we suggest one exemplary choice of a mortality model staying in the conditional independence setting introduced in the previous section.

This decomposition in a best-estimate value of the mortality-risk-related payoff and unsystematic and systematic deviations from this best-estimate value can be used to decompose the payoff $H_{T}^{n}$ in three different parts:

$$
\begin{aligned}
H_{T}^{n} & =\frac{1}{n} \sum_{j=1}^{n} g_{j}\left(\tau_{j}\right) \cdot h_{j}\left(S_{T}\right) \\
& =\underbrace{\frac{1}{n} \sum_{j=1}^{n} \mathbb{E}_{\mathcal{F}_{0}}^{\mathbb{P}^{a} \times \mathbb{P}^{f}}\left[g_{j}\left(\tau_{j}\right)\right] \cdot h_{j}\left(S_{T}\right)}_{\text {financial part }}+\underbrace{\frac{1}{n} \sum_{j=1}^{n}\left(g_{j}\left(\tau_{j}\right)-\mathbb{E}_{\mathcal{F}_{0} \vee \mathcal{G}_{T}}^{\mathbb{P}^{a} \times \mathbb{P}^{f}}\left[g_{j}\left(\tau_{j}\right)\right]\right) \cdot h_{j}\left(S_{T}\right)}_{\text {diversifiable part }}
\end{aligned}
$$




$$
\begin{aligned}
& +\underbrace{\frac{1}{n} \sum_{j=1}^{n}\left(\mathbb{E}_{\mathcal{F}_{0} \vee \mathcal{G}_{T}}^{\mathbb{P}^{a} \times \mathbb{P}^{f}}\left[g_{j}\left(\tau_{j}\right)\right]-\mathbb{E}_{\mathcal{F}_{0}}^{\mathbb{P}^{a} \times \mathbb{P}^{f}}\left[g_{j}\left(\tau_{j}\right)\right]\right) \cdot h_{j}\left(S_{T}\right)}_{\text {unhedgeable, non-diversifiable part }} \\
= & : H_{T}^{(1)}+H_{T}^{(2)}+H_{T}^{(3)} .
\end{aligned}
$$

The first part $H_{T}^{(1)}$ is a purely financial payoff that does not depend on mortality risk. This part is completely hedgeable on financial markets. For the second part $H_{T}^{(2)}$, we analyze unsystematic deviations from the population-wide mortality experience $\mathcal{G}_{T}$. This part is completely diversifiable on insurance markets. As, conditional on $\mathcal{G}_{T}$, individual deaths are independent, this term can be eliminated by increasing the portfolio size $n$ (see Theorem 3.1(a) for a proof) and is thus referred to as diversifiable risk. The risk of the last term $H_{T}^{(3)}$ cannot be eliminated. It contains systematic mortality risk and financial market risk and can be neither hedged on financial markets nor diversified by increasing the portfolio size. This hybrid part disappears if mortality rates are deterministic, that is, if there is no systematic mortality risk, see Theorem 3.1(b) for a formal proof.

In the remainder of this article, our focus is on homogeneous insurance portfolios, that is, our contracts satisfy the following assumption:

(Hom) The insured pool is homogeneous, that is, their initial age and risk preferences are equal. Their remaining lifetimes are identically distributed. Further $g_{j}\left(\tau_{j}\right)=g\left(\tau_{j}\right)$ and $h_{j}\left(S_{T}\right)=h\left(S_{T}\right)$ for $j=1,2, \ldots, n$.

Theorem 3.1 ((Non-)diversifiable mortality risk). Consider an insurance portfolio satisfying assumption (Hom). We find that:

(a) In the limit $n \rightarrow \infty$, the diversifiable part $H_{T}^{(2)}$ of the payoff $H_{T}^{n}$ converges a.s. to 0 , that is, $H_{T}^{(2)} \rightarrow 0$ a.s. (no unsystematic mortality risk).

(b) If mortality rates are deterministic (no systematic mortality risk), the last part $H_{T}^{(3)}$ of the payoff $H_{T}^{n}$ equals 0 a.s., that is, $H_{T}^{(3)}=0$ a.s.

Proof. See Appendix A.

\section{VALUATION OF HYBRID CLAIMS}

In this section, we introduce different valuation operators for hybrid life products $H_{T}^{n} \in \mathcal{H}_{T}$. In Section 4.1, we introduce the three-step method whose premium is based on the payoff decomposition (3.1). In Section 4.2, we look at the existing literature and concentrate on two so-called conditional valuation principles. The general idea of these valuation operators is to first condition on 
the financial market filtration $\mathcal{F}_{T}^{f}$ and then to apply an actuarial valuation principle on the $\mathcal{F}_{T}^{f}$-conditional payoff (see, e.g., Møller, 2002; Pelsser and Stadje, 2014). In Section 4.3, we focus on an axiomatic approach by indicating desirable properties that a valuation operator should fulfill. Finally, we show that each of the above-mentioned (hybrid) valuation operators satisfies the basic properties of a valuation operator and is both market-consistent and actuarial. In Section 4.4, we compare the three valuation operators in the case of a homogeneous insurance portfolio.

\subsection{Additive valuation: three-step method}

In Section 3, we have decomposed the payoff of a hybrid claim $H_{T}^{n} \in \mathcal{H}_{T}$ into a hedgeable part $H_{T}^{(1)}$, a diversifiable part $H_{T}^{(2)}$, and an unhedgeable and nondiversifiable part $H_{T}^{(3)}$, see (3.1). We now use this decomposition to suggest a new valuation operator for hybrid claims. This three-step valuation operator is defined as:

$$
\pi_{0}^{(3)}\left(H_{T}^{n}\right):=\pi_{0}^{f}\left(H_{T}^{(1)} \mid \mathcal{F}_{0}\right)+\pi_{0}^{a}\left(H_{T}^{(2)} \mid \mathcal{F}_{0}\right)+\pi_{0}^{n}\left(H_{T}^{(3)} \mid \mathcal{F}_{0}\right),
$$

that is, we apply a valuation operator separately for each of the three parts of the payoff. The advantage of this decomposition is its direct link to the corresponding strategies. While the application of the financial valuation on the claim $H_{T}^{(1)}$ can be justified by an underlying financial hedging strategy in a complete and arbitrage-free financial market, the valuation of the second claim $H_{T}^{(2)}$ is based on an actuarial valuation: the risks can be diversified by choosing a sufficiently large portfolio size. For the last part $H_{T}^{(3)}$, that takes into account the uncertainty of the unhedgeable and non-diversifiable part of the payoff, there exists neither a financial hedge nor a diversification strategy to reduce the inherent risk. Instead, the insurer has to provide a sufficient safety margin to account for this risk. This requires the choice of a valuation operator $\pi_{0}^{n}$ for $H_{T}^{(3)}$ that adequately reflects this neither hedgeable nor diversifiable risk. We suggest to use an Esscher valuation operator, that is, we apply an Esscher transform to the underlying risk drivers (financial risk and systematic mortality risk) to obtain the discounted value $\pi_{0}^{n}\left(H_{T}^{(3)} \mid \mathcal{F}_{0}\right)$. The Esscher valuation operator is a well-known concept in finance to, for example, valuate financial claims in incomplete financial markets (see, e.g., Chapter 9 in Cont and Tankov, 2003; Schoutens, 2003). The choice of the Esscher measure among the set of possible other equivalent martingale measures may be justified by a utility-maximizing argument (see Gerber and Shiu, 1996). The incompleteness in our setting is different from the financial applications as it stems from (systematic) mortality risk. Note that the risk of the residual part $H_{T}^{(3)}$ is different from the first two parts $H_{T}^{(1)}$ and $H_{T}^{(2)}$ as this risk has to stay in the insurer's book and cannot be reduced by hedging or diversification. Using the Esscher 
transform for the residual part is very practical as we can calibrate it using the Cost-of-Capital approach of Solvency II (see, e.g., Möhr, 2011; Zeddouk and Devolder, 2019). In insurance, it is common to charge a risk margin for the risks that stay in the insurer's books. This serves as a buffer by the shareholders to cover unhedgeable and undiversifiable risks. In the Cost-of-Capital approach, at each future time point, the insurance company's solvency capital requirements are computed. Then, the costs of this capital provision is added as a risk margin to the best-estimate insurance premium. We suggest to calibrate the Esscher valuation operator to this risk margin (see, e.g., Zeddouk and Devolder, 2019).

Note that the choice to add the premiums related to the three parts $H_{T}^{(1)}$, $H_{T}^{(2)}$, and $H_{T}^{(3)}$ is not unique and can be quite conservative. In case that there are dependencies between the different parts (e.g., between the systematic and the unsystematic mortality risk), the three-step valuation operator can serve as an upper bound on the contract value. The advantage of this upper bound and the vision behind it is that the insurer has a recipe on how to hedge the risk of the claim, that is, the insurer uses three strategies to treat the financial, actuarial, and residual risks contained in the claim.

For the Esscher transform, we follow Gerber and Shiu (1994) to find an equivalent martingale measure that takes into account the incompleteness of the insurance market and is at the same time coherent with the risk-neutral financial measure $\mathbb{Q}^{f}$. We will denote this equivalent martingale measure by $\mathbb{M}^{a} \times \mathbb{Q}^{f}$.

Therefore, we denote the contract's risk factors (e.g., financial and systematic mortality risk) by $\boldsymbol{X}_{T}:=\left(X_{T}^{(1)}, X_{T}^{(2)}, \ldots, X_{T}^{(k)}\right)^{\prime}$, where $k \in \mathbb{N}$. In the following, we will always consider $k=2$ with $X_{T}^{(1)}$ referring to systematic mortality risk and $X_{T}^{(2)}$ to financial risk.

For $\boldsymbol{\theta}:=\left(\theta^{(1)}, \theta^{(2)}\right)^{\prime} \in \mathbb{R}^{2 \times 1}$, the valuation operator for the payoff $H_{T}^{(3)}$ is defined $\mathrm{as}^{2}$

$$
\pi_{0}^{n}\left(H_{T}^{(3)}\left(\boldsymbol{X}_{T}\right)\right)=e^{-r T} \mathbb{E}_{\mathcal{F}_{0}}^{\mathbb{M}^{a} \times \mathbb{Q}^{f}}\left[H_{T}^{(3)}\left(\boldsymbol{X}_{T}\right)\right],
$$

where the measure $\mathbb{M}^{a} \times \mathbb{Q}^{f} \sim \mathbb{P}^{a} \times \mathbb{P}^{f}$ is defined by the following Esscher transform by considering a general claim $H_{T} \in \mathcal{H}_{T}$ :

$$
\mathbb{E}_{\mathcal{F}_{0}}^{\mathbb{M}^{a} \times \mathbb{Q}^{f}}\left[H_{T}\right]:=\mathbb{E}_{\mathcal{F}_{0}}^{\mathbb{P}^{a} \times \mathbb{P}^{f}}\left[H_{T} \cdot \frac{e^{-\sum_{j=1}^{2} \theta^{(j)} X_{T}^{(j)}}}{\mathbb{E}_{\mathcal{F}_{0}}^{\mathbb{P}^{a} \times \mathbb{P}^{f}}\left[e^{-\sum_{j=1}^{2} \theta^{(j)} X_{T}^{(j)}}\right]}\right] .
$$

Remark that although the sign of the third term $H_{T}^{(3)}\left(X_{T}\right)$ in the decomposition (3.1) seems to be unknown a priori, in practice, the parameter $\boldsymbol{\theta}$ of the Esscher transform will always be chosen in such a way that $\pi_{0}^{n}\left(H_{T}^{(3)}\left(\boldsymbol{X}_{T}\right)\right)$ is positive. In particular, the parameters will be chosen in another way for survival insurance products than for insurances guaranteeing only death benefits. 


\subsection{Conditional valuation}

In this section, we concentrate upon two conditional valuation principles for hybrid products, that is, the two-step method introduced in, for example, Pelsser and Stadje (2014) (see Section 4.2.1) and the conditional standard deviation principle as introduced by, for example, Møller (2002) (see Section 4.2.2).

\subsubsection{Two-step method}

In the two-step valuation principle suggested by, for example, Pelsser and Stadje (2014), the idea is an iterative procedure where, first, a conditional actuarial valuation $\pi_{0}^{a}\left(H_{T}^{n} \mid \mathcal{F}_{T}^{f} \vee \mathcal{F}_{0}\right)$ is applied, then a financial valuation operator $\pi_{0}^{f}$. Summarizing, we obtain the valuation operator

$$
\pi_{0}^{(2 a)}\left(H_{T}^{n}\right):=\pi_{0}^{f}\left(e^{r T} \cdot \pi_{0}^{a}\left(H_{T}^{n} \mid \mathcal{F}_{T}^{f} \vee \mathcal{F}_{0}\right) \mid \mathcal{F}_{0}\right) .
$$

The two-step valuation principle has many desirable properties, see our later discussion in Sections 4.3 and 4.4.

\subsubsection{Conditional standard deviation principle}

If the actuarial valuation $\pi_{0}^{a}$ is chosen to be the standard deviation principle, for example, Møller (2002) suggests an actuarial valuation operator that applies an actuarial valuation conditional on the financial market filtration $\mathcal{F}_{T}^{f}$ and defines the following valuation operator as a modified standard deviation principle, that we will refer to as "Conditional standard deviation principle":

$$
\pi_{0}^{(2 b)}\left(H_{T}^{n}\right):=e^{-r T}\left(\mathbb{E}_{\mathcal{F}_{0}}^{\mathbb{P}^{a} \times \mathbb{Q}^{f}}\left[H_{T}^{n}\right]+\frac{\alpha}{2} \sqrt{\mathbb{E}_{\mathcal{F}_{0}}^{\mathbb{P}^{a} \times \mathbb{P}^{f}}\left[\operatorname{Var}_{\mathcal{F}_{T}^{f} \vee \mathcal{F}_{0}}^{\mathbb{P a} \times \mathbb{P}^{f}}\left(H_{T}^{n}\right)\right]}\right),
$$

see Equation (5.6) in Møller (2002). The first part of $\pi_{0}^{(2 b)}\left(H_{T}^{n}\right)$ is the bestestimate valuation, while the second part adds a risk premium to parts of the payoff that cannot be hedged on financial markets.

\subsection{Axiomatic assessment of the valuation operators}

In this section, we follow an axiomatic approach and introduce desirable properties, a valuation operator has to fulfill. We start with general properties, see Definition 4.1.

Definition 4.1 (Valuation operator). A valuation operator is a function $\pi_{0}$ : $\mathcal{H}_{T} \rightarrow \mathbb{R}$ that attaches a real number to every contingent claim $H_{T}^{n} \in \mathcal{H}_{T}$. It has the following properties:

(a) Normalization: $\pi_{0}(0)=0$.

(b) Constant payoff: $\pi_{0}(x)=e^{-r T} x$ for any $x \in \mathbb{R}$.

(c) Translation invariance: $\pi_{0}\left(H_{T}^{n}+x\right)=\pi_{0}\left(H_{T}^{n}\right)+e^{-r T} x$ for any $x \in \mathbb{R}$ and $H_{T}^{n} \in \mathcal{H}_{T}$. 
(d) Positive homogeneity: $\pi_{0}\left(a \cdot H_{T}^{n}\right)=a \cdot \pi_{0}\left(H_{T}^{n}\right)$ for any $a>0$ and $H_{T}^{n} \in \mathcal{H}_{T}$.

(e) Sub-additivity: $\pi_{0}\left(G_{T}^{n}+H_{T}^{n}\right) \leq \pi_{0}\left(G_{T}^{n}\right)+\pi_{0}\left(H_{T}^{n}\right)$ for any $G_{T}^{n}, H_{T}^{n} \in \mathcal{H}_{T}$.

Property (a) normalizes the valuation operator by assigning a zero premium to a zero terminal payoff. Properties (b) and (c) assure cash invariance, that is, if any deterministic cash amount $x \in \mathbb{R}$ is added to the final payoff, the premium increases by its discounted value $e^{-r T} x$. The fact that payoffs are scalable, that is, $a$-times a given payoff leads to $a$-times the premium, is postulated by positive homogeneity (d). The last property sub-additivity acknowledges diversification effects and ensures that a portfolio's total premium can never be higher than the sum of the premia of all of its constituents. All these properties are satisfied by the financial valuation operator (2.3) and the standard deviation principle (2.5). If the standard deviation in (2.5) is replaced by the variance as a risk measure (variance principle), this valuation operator does not satisfy sub-additivity (see, e.g., Kaas et al., 2008). ${ }^{3}$

In Theorem 4.2, we show that the two-step method (Section 4.2.1), the conditional standard deviation principle (Section 4.2.2), and the three-step method (Section 4.1) define valuation operators in the sense of Definition 4.1. Further, we show that these valuation operators reduce to a financial valuation operator $\pi_{0}^{f}$ for purely financial, hedgeable risks and to an actuarial valuation operator $\pi_{0}^{a}$ for a claim that consists of unsystematic (diversifiable) mortality risk only.

Theorem 4.2. Consider an actuarial valuation principle $\pi_{0}^{a}$, a financial valuation principle $\pi_{0}^{f}$, and a valuation principle $\pi_{0}^{n}$ in the sense of Definition 4.1. The valuation operators' two-step method $\pi_{0}^{(2 a)}$ defined in (4.3), the conditional standard deviation principle $\pi_{0}^{(2 b)}$ in (4.4), and the three-step method $\pi_{0}^{(3)}$ in (4.1) are also valuation operators in the sense of Definition 4.1. The two-step method, the conditional standard deviation principle, and the three-step method are market-consistent and actuarial in the sense of Definition 2.1.

Proof. See Appendix B.

\subsection{Comparison of the (hybrid) valuation operators}

We now compare the premiums resulting from the two-step method (Section 4.2.1), the conditional standard deviation principle (Section 4.2.2), and the three-step method (Section 4.1).

Consider a homogeneous insurance portfolio (satisfying property (Hom) of Section 3), a valuation operator in the sense of Definition 4.1, and a payoff $H_{T}^{n} \in \mathcal{H}_{T}$, that is of the form

$$
H_{T}^{n}=\frac{1}{n} \sum_{j=1}^{n} g\left(\tau_{j}\right) \cdot h\left(S_{T}\right) .
$$


Exploiting the independence of financial and actuarial risks under the realworld measure $\mathbb{P}$, the homogeneity assumption and using the standard deviation principle as actuarial valuation operator $\pi_{0}^{a}$, we obtain for the two-step method (4.3):

$$
\begin{aligned}
\pi_{0}^{(2 a)}\left(H_{T}^{n}\right):= & \pi_{0}^{f}\left(e^{r T} \cdot \pi_{0}^{a}\left(H_{T}^{n} \mid \mathcal{F}_{T}^{f} \vee \mathcal{F}_{0}\right) \mid \mathcal{F}_{0}\right) \\
= & \pi_{0}^{f}\left(e^{r T} h\left(S_{T}\right) \cdot \pi_{0}^{a}\left(\frac{1}{n} \sum_{j=1}^{n} g\left(\tau_{j}\right) \mid \mathcal{F}_{T}^{f} \vee \mathcal{F}_{0}\right) \mid \mathcal{F}_{0}\right) \\
= & \pi_{0}^{f}\left(e^{r T} h\left(S_{T}\right) \cdot \pi_{0}^{a}\left(\frac{1}{n} \sum_{j=1}^{n} g\left(\tau_{j}\right) \mid \mathcal{F}_{0}\right) \mid \mathcal{F}_{0}\right) \\
= & e^{r T} \pi_{0}^{a}\left(\frac{1}{n} \sum_{j=1}^{n} g\left(\tau_{j}\right) \mid \mathcal{F}_{0}\right) \cdot \pi_{0}^{f}\left(h\left(S_{T}\right) \mid \mathcal{F}_{0}\right) \\
= & \mathbb{E}_{\mathcal{F}_{0}}^{\mathbb{P}^{a} \times \mathbb{Q}^{f}}\left[e^{-r T} h\left(S_{T}\right)\right] \\
& \cdot\left(\mathbb{E}_{\mathcal{F}_{0}}^{\mathbb{P}^{a} \times \mathbb{Q}^{f}}\left[\frac{1}{n} \sum_{j=1}^{n} g\left(\tau_{j}\right)\right]+\frac{\alpha}{2} \sqrt{\operatorname{Var}_{\mathcal{F}_{0}}^{\mathbb{P}^{a} \times \mathbb{P}^{f}}\left(\frac{1}{n} \sum_{j=1}^{n} g\left(\tau_{j}\right)\right)}\right) \\
= & e^{-r T}\left(\mathbb{E}_{\mathcal{F}_{0} \times \mathbb{Q}^{f}}^{\mathbb{P}^{a}}\left[H_{T}^{n}\right]+\frac{\alpha}{2} \mathbb{E}_{\mathcal{F}_{0}}^{\mathbb{P}^{a} \times \mathbb{Q}^{f}}\left[h\left(S_{T}\right)\right] \cdot \sqrt{\operatorname{Var}_{\mathcal{F}_{0}}^{\mathbb{P}^{a} \times \mathbb{P}^{f}}\left(\frac{1}{n} \sum_{j=1}^{n} g\left(\tau_{j}\right)\right.}\right)
\end{aligned} .
$$

The same expression is obtained by rewriting Equation (5.5) in Møller (2002). The premium depends on the value of the financial part $\mathbb{E}_{\mathcal{F}_{0}}^{\mathbb{P}^{a} \times \mathbb{Q}^{f}}\left[h\left(S_{T}\right)\right]$ and is unaffected by higher moments (e.g., the variance) of the financial payoff.

In case of the conditional standard deviation principle (4.4), we obtain

$$
\begin{aligned}
& \pi_{0}^{(2 b)}\left(H_{T}^{n}\right) \\
& =e^{-r T}\left(\mathbb{E}_{\mathcal{F}_{0} \times \mathbb{Q}^{f}}^{\mathbb{P}^{a}}\left[H_{T}^{n}\right]+\frac{\alpha}{2} \sqrt{\mathbb{E}_{\mathcal{F}_{0}}^{\mathbb{P a} \times \mathbb{P}^{f}}\left[h\left(S_{T}\right)^{2} \cdot \operatorname{Var}_{\mathcal{F}_{T}^{f} \times \mathcal{F}_{0}}^{\mathbb{P} a \mathbb{P}^{f}}\left(\frac{1}{n} \sum_{j=1}^{n} g\left(\tau_{j}\right)\right)\right]}\right) \\
& =e^{-r T}\left(\mathbb{E}_{\mathcal{F}_{0} \times \mathbb{Q}^{f}}^{\mathbb{Q}^{a}}\left[H_{T}^{n}\right]+\frac{\alpha}{2} \sqrt{\mathbb{E}_{\mathcal{F}_{0}}^{\mathbb{P a} \times \mathbb{P}^{f}}\left[h\left(S_{T}\right)^{2}\right]} \cdot \sqrt{\operatorname{Var}_{\mathcal{F}_{0}}^{\mathbb{P}^{a} \times \mathbb{P}^{f}}\left(\frac{1}{n} \sum_{j=1}^{n} g\left(\tau_{j}\right)\right)} .\right.
\end{aligned}
$$


Comparing Equations (4.5) and (4.6), we observe that the premium $\pi_{0}^{(2 b)}\left(H_{T}^{n}\right)$ takes into account the risk of the financial payoff by the term $\sqrt{\mathbb{E}_{\mathcal{F}_{0}}^{\mathbb{P a} \times \mathbb{P}^{f}}\left[h\left(S_{T}\right)^{2}\right]}$. This clearly differs from the two-step premium that depends on the fair value $\mathbb{E}_{\mathcal{F}_{0}}^{\mathbb{P}^{a} \times \mathbb{Q}^{f}}\left[h\left(S_{T}\right)\right]$ of the financial risks only. The premium of the three-step method is given by

$$
\begin{aligned}
\pi_{0}^{(3)}( & \left.H_{T}^{n}\right) \\
:= & \pi_{0}^{f}\left(H_{T}^{(1)} \mid \mathcal{F}_{0}\right)+\pi_{0}^{a}\left(H_{T}^{(2)} \mid \mathcal{F}_{0}\right)+\pi_{0}^{n}\left(H_{T}^{(3)} \mid \mathcal{F}_{0}\right) \\
= & e^{-r T}\left(\mathbb{E}_{\mathcal{F}_{0}}^{\mathbb{P}^{a} \times \mathbb{Q}^{f}}\left[H_{T}^{n}\right]+\frac{\alpha}{2} \sqrt{\mathbb{E}_{\mathcal{F}_{0}}^{\mathbb{P}^{a} \times \mathbb{P}^{f}}\left[h\left(S_{T}\right)^{2}\right]} .\right. \\
& \cdot \sqrt{\operatorname{Var}_{\mathcal{F}_{0}}^{\mathbb{P}^{a} \times \mathbb{P}^{f}}\left(\frac{1}{n} \sum_{j=1}^{n}\left(g\left(\tau_{j}\right)-\mathbb{E}_{\mathcal{F}_{0} \vee \mathcal{G}_{T}}^{\mathbb{P}^{a} \times \mathbb{P}^{f}}\left[g\left(\tau_{j}\right)\right]\right)\right)} \\
& \left.+\mathbb{E}_{\mathcal{F}_{0}}^{\mathbb{P}^{a} \times \mathbb{Q}^{f}}\left[h\left(S_{T}\right)\right] \cdot \mathbb{E}_{\mathcal{F}_{0}}^{\mathbb{M}^{a} \times \mathbb{Q}^{f}}\left[\frac{1}{n} \sum_{j=1}^{n}\left(\mathbb{E}_{\mathcal{F}_{0} \vee \mathcal{G}_{T}}^{\mathbb{P}^{a}}\left[g_{j}\left(\tau_{j}\right)\right]-\mathbb{E}_{\mathcal{F}_{0}}^{\mathbb{P}^{a} \times \mathbb{Q}^{f}}\left[g_{j}\left(\tau_{j}\right)\right]\right)\right]\right),
\end{aligned}
$$

where the measure $\mathbb{M}^{a} \times \mathbb{Q}^{f}$ is defined as the Esscher transform in (4.2). As in the case of the conditional standard deviation principle (4.6), the threestep method depends also on the risk of the financial payoff by the term $\sqrt{\mathbb{E}_{\mathcal{F}_{0}}^{\mathbb{P} \times \mathbb{P}^{f}}\left[h\left(S_{T}\right)^{2}\right]}$. The advantage of this decomposition is that we can now relate the different parts to financial risks, diversifiable risks, and residual risk that depends on systematic mortality risk and financial risk. In particular, we attribute the factor $\sqrt{\mathbb{E}_{\mathcal{F}_{0}}^{\mathbb{P a} \times \mathbb{P}^{f}}\left[h\left(S_{T}\right)^{2}\right]}$ to the term describing unsystematic mortality risk. Intuitively, this acknowledges that the unsystematic mortality risk does not allow to fully hedge financial risks. That is why the premium also depends on the real-world financial risk $\sqrt{\mathbb{E}_{\mathcal{F}_{0}}^{\mathbb{P a} \times \mathbb{P}^{f}}\left[h\left(S_{T}\right)^{2}\right.}$. This is in contrast to the two-step method (4.5) that depends on the fair value $\mathbb{E}_{\mathcal{F}_{0}}^{\mathbb{P}^{a} \times \mathbb{Q}^{f}}\left[h\left(S_{T}\right)\right]$ of the financial risks only.

\section{APPLiCATION TO PURE ENDOWMENT LIFE INSURANCE WITH PROFIT}

As an example, we consider a pure endowment life insurance contract with profit, for which the insurer pays a guaranteed fixed lump sum. The insurer has fixed a guaranteed lump sum of $1 €$ in case of life after $T$ years but no payment in case of death before time $T$. The single premium paid by the client is given by

$$
P=\frac{1}{(1+i)^{T}} \cdot T \hat{p}_{x},
$$


where $i$ and ${ }_{T} \hat{p}_{x}$ are, respectively, the technical interest rate and the survival probability chosen by the insurer. Both actuarial assumptions are prudent and chosen ex ante on a first-order basis.

The premium $P$ is invested in a (stochastic) fund $\left\{S_{t}\right\}_{t \in[0, T]}, S_{0}=P$ (see Examples 5.1-5.2). As we consider a pure endowment contract with profit, the final fund payment to the client in case of life at time $T$ contains two parts: the guaranteed amount (equal to $1 €$ ) and (possibly) a surplus participation (terminal bonus). The terminal bonus is taken to be a share $\beta \in(0,1)$ of the difference between the fund value and the premium $P$ accrued at the technical interest rate $i$ (if this difference is positive). Overall, this leads to the time- $T$ payoff

$$
h\left(S_{T}\right):=1+\beta \cdot \max \left(S_{T}-P(1+i)^{T}, 0\right) .
$$

We now consider a portfolio of $n$ homogeneous (see property (Hom) in Section 3) endowment insurance contracts. The pure endowment life insurance contract with profit is a hybrid payoff $H_{T}^{n} \in \mathcal{H}_{T}$, where the payoff to client $j \in\{1,2, \ldots, n\}$ is given by

$$
H_{T}(j)=g\left(\tau_{j}\right) \cdot h\left(S_{T}\right)=\mathbb{1}_{\left\{\tau_{j}>T\right\}} \cdot\left(1+\beta \cdot \max \left(S_{T}-P(1+i)^{T}, 0\right)\right) .
$$

The average payment to one client is then given by $H_{T}^{n}:=\frac{1}{n} \sum_{j=1}^{n} H_{T}(j)$. For the financial fund, we consider two possible dynamics: a risk-free bank account (Example 5.1) and a Black-Scholes model (Example 5.2).

Example 5.1 (Fund dynamics 1: Bank account). The premium $P$ is invested in a risk-free bank account with constant interest rate $r \geq i$, that is, $S_{t}=P \cdot e^{r t}$. In this case, the financial payoff (5.2) of the pure endowment life insurance contract is given by

$$
h\left(S_{T}\right):=1+\beta P \cdot\left(e^{r T}-(1+i)^{T}\right)
$$

The expected present value of this payoff is

$$
\mathbb{E}_{\mathcal{F}_{0}}^{\mathbb{P}^{a} \times \mathbb{Q}^{f}}\left[e^{-r T} h\left(S_{T}\right)\right]=e^{-r T}\left(1+\beta P \cdot\left(e^{r T}-(1+i)^{T}\right)\right) .
$$

For later purposes (namely, the calculations of the premium in three-step method and the conditional standard deviation principle), we notice that

$$
\sqrt{\mathbb{E}_{\mathcal{F}_{0}}^{\mathbb{P} \times \mathbb{P}^{f}}\left[h\left(S_{T}\right)^{2}\right]}=1+\beta P \cdot\left(e^{r T}-(1+i)^{T}\right) .
$$

Example 5.2 (Fund dynamics 2: Black-Scholes model). Apart from the riskfree bank account, the financial market consists of one $\mathcal{F}_{t}^{f}$-adapted risky asset with $\mathbb{P}^{f}$-dynamics

$$
\mathrm{d} S_{t}=\mu_{S} S_{t} \mathrm{~d} t+\sigma_{S} S_{t} \mathrm{~d} W_{t}, \quad S_{0}=P,
$$

where $W_{t}$ is a $\mathbb{P}^{f}$-Brownian motion, which models the fund dynamics. We assume that there exists an equivalent martingale measure $\mathbb{Q}^{f}$ and we can 
change to the $\mathbb{Q}^{f}$-Brownian motion $W_{t}^{\mathbb{Q}^{f}}:=W_{t}+\frac{\mu_{S}-r}{\sigma_{S}} \cdot t$. For the payoff (5.2) of the pure endowment life insurance contract, we obtain

$$
\begin{aligned}
\mathbb{E}_{\mathcal{F}_{0}}^{\mathbb{P}^{a} \times \mathbb{Q}^{f}}\left[e^{-r T} h\left(S_{T}\right)\right] & =e^{-r T}\left(1+\beta P \cdot\left(\Phi\left(d_{1}\left(r, \sigma_{S}\right)\right)-G \cdot \Phi\left(d_{2}\left(r, \sigma_{S}\right)\right)\right)\right) \\
& =: e^{-r T} K\left(r, \sigma_{S}\right),
\end{aligned}
$$

where $d_{1}(v, \sigma):=\left(\left(v+\frac{\sigma^{2}}{2}\right) T-\ln (G)\right) /(\sigma \sqrt{T}), d_{2}(v, \sigma):=d_{1}(v, \sigma)-\sigma \sqrt{T}$, $G:=(1+i)^{T}$ and $\Phi$ denotes the standard normal cumulative distribution function. As in Example 5.1, we compute

$$
\sqrt{\mathbb{E}_{\mathcal{F}_{0}}^{\mathbb{P} a \mathbb{P}^{f}}\left[h\left(S_{T}\right)^{2}\right]}=\sqrt{1+2 \beta P \cdot K\left(\mu_{S}, \sigma_{S}\right)+\beta^{2} P^{2} \cdot K_{2}\left(\mu_{S}, \sigma_{S}\right)},
$$

where

$K_{2}\left(\mu_{S}, \sigma_{S}\right):=G^{2} \Phi\left(d_{2}\left(\mu_{S}, \sigma_{S}\right)\right)-2 G e^{\mu_{S} T} \Phi\left(d_{1}\left(\mu_{S}, \sigma_{S}\right)\right)+e^{\left(2 \mu_{S}+\sigma_{S}^{2}\right) T} \Phi\left(d_{3}\left(\mu_{S}, \sigma_{S}\right)\right)$

with $d_{3}(v, \sigma):=d_{1}(v, \sigma)+\sigma \sqrt{T}$.

To compute the premium of the pure endowment life insurance contract, we still need to model the systematic mortality risk. We consider a stochastic mortality intensity $\left\{\lambda_{t}\right\}_{t \in[0, T]}$, adapted to the filtration $\mathcal{G}$. The survival probability of individual $j$ with age $x$ is abbreviated by

$$
{ }_{T-t} p_{x}^{j}:=\mathbb{P}\left(\tau_{j} \geq T \mid \tau_{j}>t, \mathcal{F}_{t}\right)=\mathbb{1}_{\left\{\tau_{j}>t\right\}} \cdot \mathbb{E}_{\mathcal{F}_{t}}^{\mathbb{P}}\left[e^{-\int_{t}^{T} \lambda_{s} \mathrm{~d} s}\right] .
$$

We notice that both the remaining lifetimes and the stochastic mortality intensity process will depend upon the age $x$. Although this could have been stressed in the notation, we prefer to omit the dependence in the notation for notational convenience. For calculating the best-estimate survival probabilities, we choose a stochastic model for the mortality intensity $\left\{\lambda_{t}\right\}_{t \in[0, T]}$. Indeed, in Example 5.3, we introduce an Ornstein-Uhlenbeck (OU) mortality intensity.

Example 5.3 (Mortality model: Ornstein-Uhlenbeck intensity). As in, for example, Dahl and Møller (2006), Luciano and Vigna (2008), Luciano et al. (2012), we model systematic mortality risk by the stochastic mortality intensity following an Ornstein-Uhlenbeck process, that is,

$$
\mathrm{d} \lambda_{t}=\mu_{\lambda} \lambda_{t} \mathrm{~d} t+\sigma_{\lambda} \mathrm{d} W_{t}^{\lambda}, \quad \lambda_{0}>0,
$$

where we assume that $W_{t}^{\lambda}$ is independent of the financial risk filtration $\mathcal{F}_{t}^{f}$ and the parameters satisfy the usual conditions (see, e.g., Ikeda and Watanabe, 2014). From this, we find that the integrated intensity $\int_{t}^{T} \lambda_{s} \mathrm{~d} s$ is normally distributed with 


$$
\begin{aligned}
m_{t, T} & :=\mathbb{E}_{\mathcal{F}_{t}}^{\mathbb{P}^{a} \mathbb{P}^{f}}\left[\int_{t}^{T} \lambda_{s} \mathrm{~d} s\right]=\lambda_{t} \cdot \zeta(t, T), \\
s_{t, T}^{2} & :=\operatorname{Var}_{\mathcal{F}_{t}}^{\mathbb{P}^{a} \times \mathbb{P}^{f}}\left(\int_{t}^{T} \lambda_{s} \mathrm{~d} s\right)=\frac{\sigma_{\lambda}^{2}}{\mu_{\lambda}^{2}}\left(\frac{\mu_{\lambda}}{2} \cdot \zeta^{2}(t, T)-\zeta(t, T)+(T-t),\right.
\end{aligned}
$$

where $\zeta(t, T):=\left(e^{\mu_{\lambda}(T-t)}-1\right) / \mu_{\lambda}$. We can use this to obtain the survival probability (5.5) of individual $j$ with an initial age $x$ :

$$
T-t p_{x}^{j}:=\mathbb{1}_{\left\{\tau_{j}>t\right\}} \cdot \mathbb{E}_{\mathcal{F}_{t}}^{\mathbb{P}^{a} \times \mathbb{P}^{f}}\left[e^{-\int_{t}^{T} \lambda_{s} \mathrm{~d} s}\right]=\mathbb{1}_{\left\{\tau_{j}>t\right\}} \cdot e^{-m_{t, T}+\frac{1}{2} s_{t, T}^{2}} .
$$

The computations of the different parts of the three-step premium for an Ornstein-Uhlenbeck mortality intensity, as introduced in Example 5.3, can be found in Appendix C. From these calculations, it is clear that they are essentially based on the laws (conditional upon $\mathcal{F}_{t}$ ) of the random variables $\left(-\int_{t}^{T} \lambda_{s} \mathrm{~d} s\right)$ for the mortality modeling and $\ln \left(S_{T} / S_{t}\right)$ in case of the financial risk as modeled in Example 5.2. In the following, we refer to these risk factors as $X_{T}^{(1)}$ and $X_{T}^{(2)}$.

\section{EXTENSION TO GENERAL LIFE CONTINGENCIES AND SECURITIZATION}

The valuation procedure proposed in Section 5 for pure endowment life insurance contracts can be extended to other kinds of life contingencies and to life securitization products.

\subsection{General life contingencies}

So far, we have considered retirement products with a single payoff at a fixed maturity time $T$. If we want to generalize this approach to term insurance or annuity products, we have to introduce multi-period cash flows. The payoff of a general life product is then a vector of random variables representing the successive potential payoffs of the product at times $t=1, \ldots, T$. Generalizing (2.1), these cash flows - paid by the insurer to the various policyholders belonging to a pool - are introduced as

$$
\boldsymbol{H}^{n}:=\left(H_{1}^{n}, H_{2}^{n}, \ldots, H_{T}^{n}\right),
$$

where $H_{t}^{n}$ is the time- $t$ random payoff given by:

$$
H_{t}^{n}=\frac{1}{n} \sum_{j=1}^{n} g_{j, t}\left(\tau_{j}\right) \cdot h_{j, t}\left(\left(S_{s}\right)_{0 \leq s \leq t}\right), \quad t=1, \ldots, T .
$$


In particular, for a homogeneous pool of policyholders: $H_{t}^{n}=\frac{1}{n} \sum_{j=1}^{n} g_{t}\left(\tau_{j}\right)$. $h_{t}\left(\left(S_{s}\right)_{0 \leq s \leq t}\right)$, for $t=1, \ldots, T$. We can model various classical life insurance products this way:

Example 1. Pure endowment

A pure endowment contract corresponds to the special case $h_{t}\left(\left(S_{s}\right)_{0 \leq s \leq t}\right)=1$ and

$$
g_{t}\left(\tau_{j}\right)=0, \quad t=1,2, \ldots, T-1, \quad \text { and } \quad g_{T}\left(\tau_{j}\right)=\mathbb{1}_{\left\{\tau_{j}>T\right\}},
$$

see also Section 5.

Example 2. Term insurance

A term insurance contract (payment of one unit at the end of the year $t$ in case of death between age $x+t-1$ and age $x+t$ ) is characterized by $h_{t}\left(\left(S_{s}\right)_{0 \leq s \leq t}\right)=1$ and

$$
g_{t}\left(\tau_{j}\right)=\mathbb{1}_{\left\{t-1<\tau_{j} \leq t\right\}}, \quad t=1,2, \ldots, T .
$$

We can valuate this product using the three-step method. The first part corresponds to the usual expected present value:

$$
\pi_{0}^{f}\left(H_{T}^{(1)} \mid \mathcal{F}_{0}\right)=\sum_{t=1}^{T} e^{-r t} \cdot{ }_{t-1} p_{x} \cdot q_{t},
$$

where ${ }_{t} p_{x}:=\mathbb{P}\left(\tau_{j} \geq t\right)=\mathbb{E}_{\mathcal{F}_{0}}^{\mathbb{P}}\left[e^{-\int_{0}^{t} \lambda_{s} \mathrm{~d} d}\right]$ and $q_{t}:=\mathbb{P}\left(t-1<\tau_{j} \leq t\right)={ }_{t} p_{x}-{ }_{t-1} p_{x}$. For the diversifiable part $H_{T}^{(2)}$, we use the standard deviation principle (2.5) accruing all payments to maturity at the risk-free rate $r$ :

$$
\begin{aligned}
\pi_{0}^{a} & \left(H_{T}^{(2)} \mid \mathcal{F}_{0}\right) \\
& =e^{-r T} \frac{\alpha}{2} \sqrt{\operatorname{Var}_{\mathcal{F}_{0}}^{\mathbb{P}^{a} \times \mathbb{P}^{f}}\left(\frac{1}{n} \sum_{t=1}^{T} e^{r(T-t)}\left(\left(N_{t-1}-N_{t}\right)-n \cdot\left(e^{-\int_{0}^{t-1} \lambda_{s} \mathrm{~d} s}-e^{-\int_{0}^{t} \lambda_{s} \mathrm{~d} s}\right)\right)\right)} .
\end{aligned}
$$

For the non-diversifiable and unhedgeable part $H_{T}^{(3)}$, we introduce the normally distributed risk factors $X_{t}^{(1)}:=-\int_{0}^{t} \lambda_{s} \mathrm{~d} s$ and denote the covariance matrix of $\left(X_{t-1}^{(1)}, X_{t}^{(1)}\right)$ by $\boldsymbol{\Sigma}^{t}$. Then we find by applying the Esscher transform: 


$$
\begin{aligned}
& \pi_{0}^{n}\left(H_{T}^{(3)} \mid \mathcal{F}_{0}\right)
\end{aligned}
$$

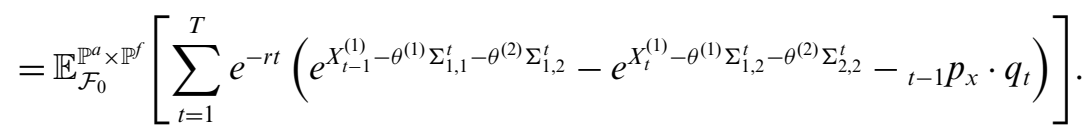

Again, there is an explicit hedging and diversification strategy behind the first two parts of the premium.

\section{Example 3. Annuity}

An annuity product (payment of one unit at the end of each year in case of survival of the policyholder, until the maximum age $x+T)$ is characterized by $h_{t}\left(\left(S_{s}\right)_{0 \leq s \leq t}\right)=1$ and

$$
g_{t}\left(\tau_{j}\right)=\mathbb{1}_{\left\{\tau_{j}>t\right\}}, \quad t=1,2, \ldots, T .
$$

An application of the three-step method works similar to Example 2.

All these examples can be generalized to their analogs with profit sharing, where the fund is either based on Example 5.1 or 5.2.

\subsection{Securitization products}

We can also adapt the methodology for the pricing of life securitization products such as survival or CAT bonds or swaps. For instance, let us consider a bond with fixed coupon and with a principal at risk, linked to a survival or mortality condition on a reference population. We denote by $T$ the maturity of the bond, by $K$ the nominal, and by $c$ the coupon rate. Then, the bond's cash flow looks as follows:

$$
\begin{aligned}
\mathrm{CF}_{t} & =c \cdot K, \quad t=1,2, \ldots, T, \\
\mathrm{CF}_{T} & =c \cdot K+H_{T}^{n},
\end{aligned}
$$

where $H_{T}^{n}$ is based on a stochastic index linked to the realized longevity or mortality of the reference population at maturity $T$.

In this case, the random variable $H$ is no more linked to the individual situation of each policyholder (individual function $g_{j}$ for each insured as in Section 6.1 above) but to the global behavior of the population. Then, instead of considering individual cash flows depending on each remaining lifetime through the functions $g_{j}$ (formula (2.1)), we have to model a random variable depending globally on all the lifetimes simultaneously. Formally, instead of considering a maturity risk of the form $H_{T}^{n}=\frac{1}{n} \sum_{j=1}^{n} g\left(\tau_{j}\right) \cdot h\left(S_{T}\right)$, we can model a global cash flow by:

$$
H_{T}^{n}=G\left(\tau_{1}, \tau_{2}, \ldots, \tau_{n}\right) \cdot h\left(S_{T}\right) .
$$


Example 1. Survivor swap

Without coupon $(c=0)$ and with a principal given by :

$$
H_{T}^{n}=K \cdot\left(I_{n}(x, T)-{ }_{T} p_{x}\right),
$$

where

$$
\begin{array}{ll}
I_{n}(x, T) & \begin{array}{l}
\text { realized survival rate at age } x+T \text { of a pool of } n \text { policyholders } \\
\text { initially aged } x,
\end{array} \\
{ }_{T} p_{x} & \begin{array}{l}
\text { ex ante probability of survival between ages } x \text { and } \\
\\
x+T \text { (chosen life table), }
\end{array}
\end{array}
$$

we obtain the payoff of a survivor swap. More formally, the realized survival rate is given by $I_{n}(x, T)=N_{T} / n$. The decomposition of the three-step method is very similar to the pure endowment contracts in Section 5 and therefore omitted.

\section{Example 2. CAT bonds}

A CAT bond is considered to be a bond with maturity $T$ and nominal $K$ that annually pays financial coupons at a rate $c$ (see, e.g., Lin and Cox, 2008; Tsai and Tzeng, 2013). The repayment of the principal $K$ at maturity is at risk, that is, the issuer of the bond will repay less to the investors in order to recuperate part of his mortality-risk-related liabilities. To link the payoff to mortality risk, we consider a reference population of size $n$ with initial 1-year death probability $q_{0}$. Following Lin and Cox (2008), we introduce the payoff of the 2003 Swiss Re mortality bond with a maturity of $T=3$ years. The loss of principal is linked to how far the realized mortality $q:=\max _{t=1,2,3} q_{t}$, where

$$
q_{t}:=\frac{1}{n} \sum_{j=1}^{n} \mathbb{1}_{\left\{t-1<\tau_{j} \leq t\right\}}, \quad t=1,2,3,
$$

deviates from $q_{0}$. The bond's cash flow looks as in (6.2) with $H_{T}^{n}=K \cdot l$, where the loss is determined as the following function $l(q)$, which is therefore a function of $\tau_{j}$ for all $j=1, \ldots, n$ :

$$
l(q)= \begin{cases}1, & \text { if } q \leq 1.3 \cdot q_{0} \\ \frac{1.5 \cdot q_{0}-q}{0.2 \cdot q_{0},} & \text { if } 1.3 \cdot q_{0}<q \leq 1.5 \cdot q_{0} \\ 0, & \text { if } q \geq 1.5 \cdot q_{0}\end{cases}
$$

We can first decompose the CAT bond payoff in a bond payoff with nominal $K$ and coupon rate $c$ and a mortality-related payoff $H_{T}^{n}$, which is clearly a payoff of the form (6.3) with $G\left(\tau_{1}, \tau_{2}, \ldots, \tau_{n}\right)=l$ and $h\left(S_{T}\right)=K$.

The coupon part of the CAT bond payoff is a purely financial payoff with present value $c K \sum_{t=1}^{T} e^{-r t}$. To valuate the remaining payoff $H_{T}^{n}$, we can use the 
three-step decomposition (4.1). The value of the hedgeable part corresponds to the expected present value:

$$
\pi_{0}^{f}\left(H_{T}^{(1)} \mid \mathcal{F}_{0}\right)=e^{-r T} K \cdot \mathbb{E}_{\mathcal{F}_{0}}^{\mathbb{P}^{a} \times \mathbb{Q}^{f}}[l(q)] .
$$

For the diversifiable part, we need to account for the risk that the $\mathcal{G}_{T^{-}}$ conditional payoff deviates from the true payoff, that is,

$$
\pi_{0}^{a}\left(H_{T}^{(2)} \mid \mathcal{F}_{0}\right)=K \cdot \pi_{0}^{a}\left(l(q)-\mathbb{E}_{\mathcal{F}_{0} \vee \mathcal{P}_{T}}^{\mathbb{P}^{p}}[l(q)]\right) .
$$

For the residual part, we get

$$
\pi_{0}^{n}\left(H_{T}^{(3)} \mid \mathcal{F}_{0}\right)=K \cdot \pi_{0}^{n}\left(\mathbb{E}_{\mathcal{F}_{0} \vee \mathcal{G}_{T}}^{\mathbb{P}^{a} \mathbb{P}^{f}}[l(q)]-\mathbb{E}_{\mathcal{F}_{0}}^{\mathbb{P}^{a} \times \mathbb{P}^{f}}[l(q)]\right) .
$$

To explicitly calculate the expressions, we can proceed in a similar way as in Example 2 in Section 6.1.

\section{NUMERICAL iLlUSTRATIONS}

To illustrate the different valuation operators, we return to the pure endowment insurance contracts from Section 5 and first choose a base-case parameter set. Then, we analyze the effect of different parameters on the premium. We choose a portfolio of $n$ pure endowment insurance contracts with profit that have a time to maturity of $T=15$ for a cohort of 65 -year-old females.

\subsection{Parameter choice}

We first compute the actuarial, prudent premium (5.1). We follow the rules recommended by the Belgian regulatory authorities (CBFA) and consider a prudent mortality table computed according to the Makeham model with parameters fixed by the Royal Decree. ${ }^{4}$ We choose the technical interest rate $i=0.9 \%$. The actuarial, prudent premium can then be computed as

$$
P=\frac{1}{(1+i)^{T}} \cdot{ }_{T} \hat{p}_{x}=\frac{1}{1.009^{15}} \cdot{ }_{1} \hat{p}_{65} \cdot{ }_{1} \hat{p}_{66} \cdots{ }_{1} \hat{p}_{79} \approx 0.663743 .
$$

For the best-estimate survival probabilities ${ }_{T} p_{x}$, we calibrate the parameters of the OU process for systematic mortality risk (see Example 5.3) to mortality rates of Belgian females (1930 cohort) obtained from the human mortality database (HMD). ${ }^{5}$ We choose the 1900 cohort and obtain survival probabilities ${ }_{T} p_{65}^{\mathrm{HMD}}$ for $T=1,2, \ldots, 30$ from the HMD. Following, for example, Chen and Vigna (2017), we minimize least-square differences to the survival probabilities of our OU mortality model. The initial mortality intensity can be 
TABLE 1

MODEL PARAMETERS FOR FINANCIAL AND SYSTEMATIC MORTALITY RISK.

\begin{tabular}{lcccccccc}
\hline \hline & \multicolumn{3}{c}{ Black-Scholes } & & \multicolumn{3}{c}{ OU process } \\
\cline { 2 - 4 } \cline { 7 - 8 } Parameters & $r$ & $\mu_{S}$ & $\sigma_{S}$ & & $\mu_{\lambda}$ & $\sigma_{\lambda}$ & $\lambda_{0}$ \\
Values & $2 \%$ & $6 \%$ & 0.20 & & 0.113826 & 0.002990 & 0.015030 \\
\hline \hline
\end{tabular}

observed from the mortality table and is given by $\lambda_{0}=0.015030$. The remaining parameters are obtained via

$$
\left(\mu_{\lambda}, \sigma_{\lambda}\right)=\min _{\mu_{\lambda}, \sigma_{\lambda}} \sum_{T=1}^{30}\left(e^{-m_{0, T}+\frac{1}{2} s_{0, T}^{2}}-{ }_{T} p_{65}^{\mathrm{HMD}}\right)^{2},
$$

see Table 1. For the financial market, we choose a constant risk-free interest rate $r=2 \%$ and Black-Scholes parameters $\mu_{S}=6 \%, \sigma_{S}=0.20$.

We compare the premium in case the funds are invested in a bank account (Example 5.1, $\beta=0.9500$ ) and a risky fund (Example 5.2, $\beta=0.4019$ ), respectively. The $\beta$-values are chosen such that the best-estimate premium (no risk premiums) for the pure endowment insurance contract with maturity $T=15$ is (in both cases) given by:

$$
P^{\mathrm{BE}}=\mathbb{E}_{\mathcal{F}_{0}}^{\mathbb{P}^{a} \times \mathbb{Q}^{f}}\left[H_{T}^{n}\right]=\pi_{0}^{f}\left(H_{T}^{(1)} \mid \mathcal{F}_{0}\right) \approx 0.471608 .
$$

For the Esscher transform, we can deduce for the two risk factors $X_{T}^{(1)}:=$ $-\int_{0}^{T} \lambda_{s} \mathrm{~d} s$ and $X_{T}^{(2)}:=\ln \left(S_{T} / S_{0}\right)$ that the means of $\left(X_{T}^{(1)}, X_{T}^{(2)}\right)$ are given by $\boldsymbol{\mu}:=\left(\mu^{(1)}, \mu^{(2)}\right)^{\prime}=\left(-m_{0, T},\left(\mu_{S}-\sigma_{S}^{2} / 2\right) T\right)^{\prime}$, and that the covariance matrix is composed as follows: $\Sigma_{1,1}=s_{0, T}^{2}, \Sigma_{1,2}=\Sigma_{2,1}=0$ and $\Sigma_{2,2}=\sigma_{S}^{2} T$. In the case of a deterministic financial payoff (a bank account), however, the financial risk does not depend on the risk factor $X_{T}^{(2)}$.

\subsection{Numerical results}

We can now apply the different valuation operators to the pure endowment insurance contract. To allow for an easy comparison, we use the best-estimate value of the contract (7.1) as $100 \%$ benchmark. A premium of $110 \%$ means that there is a $10 \%$ risk margin on top of the best-estimate premium.

\subsubsection{Comparison to the conditional valuation principles}

Next, we compare the three-step method to the two-step method by Pelsser and Stadje (2014) $\left(\pi_{0}^{(2 a)}\left(H_{T}^{n}\right)\right)$ and the conditional standard deviation principle by Møller (2002) $\left(\pi_{0}^{(2 b)}\left(H_{T}^{n}\right)\right)$. We now model systematic mortality risk as described 
TABLE 2

PREMIUM OF A PURE ENDOWMENT INSURANCE CONTRACT WITH A PARTICIPATION SHARE $\beta=0.95$, MATURITY $T=15, \alpha=0.3$ AND THE PARAMETERS FROM TABLE 1 - CASE OF A BANK ACCOUNT AND A POOL SIZE OF $n=100$.

\begin{tabular}{|c|c|c|c|c|c|c|}
\hline & \multicolumn{2}{|c|}{ Conditional valuation } & \multicolumn{3}{|c|}{ Three-step method } & \multirow[b]{2}{*}{$\pi_{0}^{(3)}\left(H_{T}^{n}\right)(\%)$} \\
\hline & $\pi_{0}^{(2 a)}\left(H_{T}^{n}\right)(\%)$ & $\pi_{0}^{(2 b)}\left(H_{T}^{n}\right)(\%)$ & $\pi_{0}^{f}\left(H_{T}^{(1)}\right)(\%)$ & $\pi_{0}^{a}\left(H_{T}^{(2)}\right)(\%)$ & $\pi_{0}^{n}\left(H_{T}^{(3)}\right)(\%)$ & \\
\hline$n=100$ & 103.46 & 103.46 & 100.00 & 1.28 & 4.32 & 105.60 \\
\hline$n=500$ & 103.26 & 103.26 & 100.00 & 0.57 & 4.32 & 104.90 \\
\hline$n=2000$ & 103.22 & 103.22 & 100.00 & 0.29 & 4.32 & 104.61 \\
\hline$n=\infty$ & 103.21 & 103.21 & 100.00 & 0.00 & 4.32 & 104.32 \\
\hline
\end{tabular}

in Example 5.3 using the parameters from Table 1. For the standard deviation principle, we choose a risk aversion parameter $\alpha=0.3$. For the financial market risk, we use $\theta^{(2)}=\left(\mu_{S}-r\right) / \sigma_{S}^{2}=1.0$, consistent with the change of measure from the real-world measure $\mathbb{P}^{f}$ to the risk-neutral measure $\mathbb{Q}^{f}$. To get reasonable values for the systematic mortality risk parameter $\theta^{(1)}$, we follow Zeddouk and Devolder (2019) and compute the risk margin for systematic mortality risk resulting from a Solvency II Cost-of-Capital approach with a cost of capital rate of $6 \%$. The parameter $\theta^{(1)}$ is then chosen such that the third part of the three-step premium equals the Solvency II risk margin for systematic mortality risk. With the pure endowment insurance contract and the parameters from Table 1 , we obtain $\theta^{(1)}=-0.945$.

In Tables 2 and 3, we present the premiums of the pure endowment insurance contract with profit for the two-step method $\pi_{0}^{(2 a)}\left(H_{T}^{n}\right)$, the conditional standard deviation principle $\pi_{0}^{(2 b)}\left(H_{T}^{n}\right)$, and the three-step method $\pi_{0}^{(3)}\left(H_{T}^{n}\right)$. In case of the three-step method, we also provide the three parts of the premium: financial, hedgeable risk, diversifiable risk, and unhedgeable, non-diversifiable risk. To make the results easily comparable, the financial part $\pi_{0}^{f}\left(H_{T}^{(1)} \mid \mathcal{F}_{0}\right)=$ 0.471608 is set to $100 \%$. Tables 2 and 3 differ in the way the premium is invested on financial markets. While Table 2 considers a bank account (see Example 5.1), Table 3 uses a risky stock (see Example 5.2). We choose the bonus share $\beta$ in a way that the financial part $\pi_{0}^{f}\left(H_{T}^{(1)} \mid \mathcal{F}_{0}\right)=0.471608$ is the same in both tables, that is, $\beta=0.4019$ for the risky stock and $\beta=0.9500$ for the bank account.

Comparing both tables, we observe that the two-step method $\pi_{0}^{(2 a)}\left(H_{T}^{n}\right)$ asks for the same insurance premium irrespective of the financial investment. The reason for this observation is the fact that the two-step premium depends on the risk-neutral price of the financial risk only. For all the other valuation operators, the premium in case of a stock investment is higher than in the case of a bank account investment. The advantage of the three-step method is the possibility to analyze the reason for this difference. Comparing Tables 2 and 3, we 
TABLE 3

PREMIUM OF A PURE ENDOWMENT INSURANCE CONTRACT WITH A PARTICIPATION SHARE $\beta=0.4019$, MATURITY $T=15, \alpha=0.3$ AND THE PARAMETERS FROM TABLE 1 - CASE OF A RISKY STOCK AND A POOL SIZE OF $n=100$.

\begin{tabular}{lcccccccc}
\hline \hline & \multicolumn{2}{c}{ Conditional valuation } & & \multicolumn{3}{c}{ Three-step method } & & \\
& & $\pi_{0}^{(2 a)}\left(H_{T}^{n}\right)(\%)$ & $\pi_{0}^{(2 b)}\left(H_{T}^{n}\right)(\%)$ & & $\pi_{0}^{f}\left(H_{T}^{(1)}\right)(\%)$ & $\pi_{0}^{a}\left(H_{T}^{(2)}\right)(\%)$ & $\pi_{0}^{n}\left(H_{T}^{(3)}\right)(\%)$ & $\pi_{0}^{(3)}\left(H_{T}^{n}\right)(\%)$ \\
\hline$n=100$ & 103.46 & 104.57 & & 100.00 & 1.69 & 4.32 & 106.01 \\
$n=500$ & 103.26 & 104.31 & & 100.00 & 0.76 & 4.32 & 105.08 \\
$n=2000$ & 103.22 & 104.26 & & 100.00 & 0.38 & 4.32 & 104.71 \\
$n=$ & $\infty$ & 103.21 & 104.24 & & 100.00 & 0.00 & 4.32 & 104.32 \\
\hline \hline
\end{tabular}

observe that the difference stems from the second, diversifiable part $\pi_{0}^{a}\left(H_{T}^{(2)}\right)$ of the premium. For the diversifiable risk $H_{T}^{(2)}$, it is interesting to compare Tables 2 (risk-free payoff) and 3 (risky payoff). In this case, the uncertainty of the mortality risk does also not allow to fully hedge the financial risks (even in a complete financial market). In case of the much riskier payoff in Table 3, the risk premium for unsystematic mortality risk is higher than for the constant payoff (Table 2). If the portfolio size is increased, the unsystematic mortality risk is fully diversified and $\pi_{0}^{a}\left(H_{T}^{(2)}\right)=0.00 \%$.

\subsubsection{Systematic and unsystematic mortality risk}

We can further analyze the effect of portfolio size $n$ on the insurance premium. Figure 1 presents on the left-hand side the different parts of the three-step premium. As a reference value $(100 \%)$, we again choose the contract's bestestimate value $\pi_{0}^{f}\left(H_{T}^{(1)} \mid \mathcal{F}_{0}\right)=0.471608$; we cut this graph at $90 \%$. On the right-hand side, the premium is compared to the two-step method and the conditional standard deviation principle. The parameter set used is the same as in Table 3 with an investment in a risky stock. We observe that the hedgeable part and the premium part for residual risk are unaffected by portfolio size. With increasing portfolio size $n$, the premium for the diversifiable part is reduced. However, even in case of a portfolio size of $n=1000$, this part of the premium contributes a significant part of the overall premium. In the limit $n \rightarrow \infty$, we arrive at a risk premium for a fully diversified insurance portfolio. In other words, there is no unsystematic mortality risk and the payoff $H_{T}^{(2)}$ of the three-step method is zero.

Lastly, we want to have a look how a different allocation between systematic and unsystematic mortality risks affects the insurance premium. We therefore have a look at three possible scenarios:

- scenario 1: A pool size of $n=50$ and the parameter set from Table 3 except for $\mu_{l}=0.113196, \sigma_{l}=0.002754$, and $\lambda_{0}=0.015030$. 
TABLE 4

PREMIUM OF A PURE ENDOWMENT INSURANCE CONTRACT WITH A PARTICIPATION SHARE $\beta=0.4019$, MATURITY $T=15, \alpha=0.3$ AND THE PARAMETERS FROM TABLE 1 - CASE OF A RISKY STOCK AND DIFFERENT SCENARIOS

\begin{tabular}{lcccccccc}
\hline \hline & \multicolumn{2}{c}{ Conditional valuation } & & \multicolumn{3}{c}{ Three-step method } & & \\
\cline { 2 - 3 } & & $\pi_{0}^{(2 a)}\left(H_{T}^{n}\right)(\%)$ & $\pi_{0}^{(2 b)}\left(H_{T}^{n}\right)(\%)$ & $\pi_{0}^{f}\left(H_{T}^{(1)}\right)(\%)$ & $\pi_{0}^{a}\left(H_{T}^{(2)}\right)(\%)$ & $\pi_{0}^{n}\left(H_{T}^{(3)}\right)(\%)$ & $\pi_{0}^{(3)}\left(H_{T}^{n}\right)(\%)$ \\
\hline Scenario 1 & 103.46 & 104.57 & & 100.00 & 2.41 & 3.62 & \\
Scenario 2 & 103.46 & 104.57 & & 100.00 & 1.69 & 4.32 & & 106.03 \\
Scenario 3 & 103.46 & 104.57 & & 100.00 & 0.53 & 4.94 & & 105.47 \\
\hline \hline
\end{tabular}
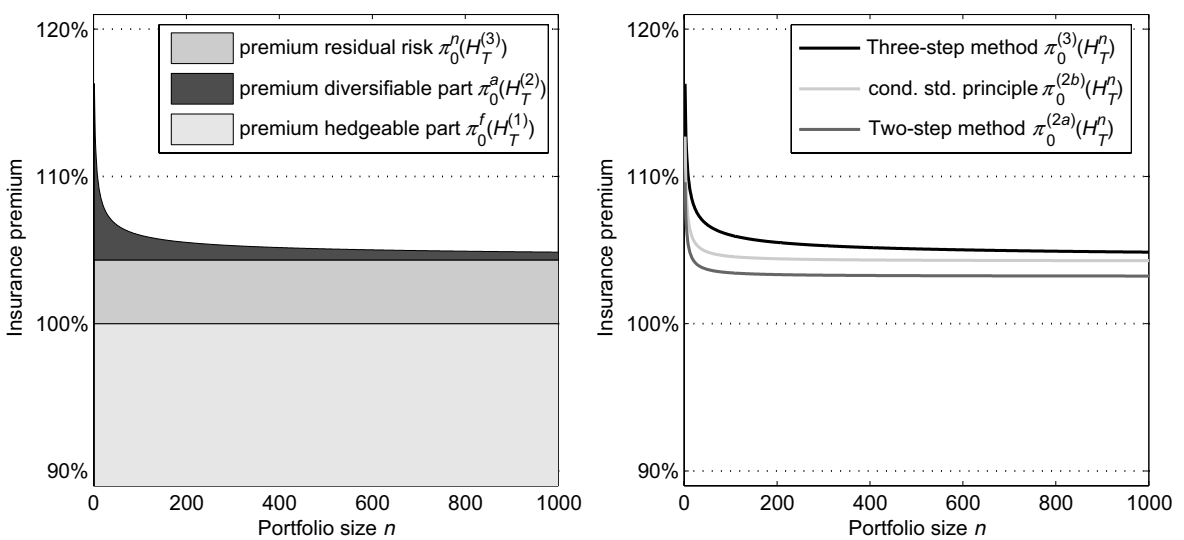

FIGURE 1: The different parts of the three-step insurance premium as a function of the portfolio size $n$ (left-hand side) and a comparison of the three-step, the two-step, and the conditional standard deviation premium (right hand side). We choose the parameters from Table 3.

- scenario 2: A pool size of $n=100$, the parameter set from Table 3 (for systematic mortality risk, this implies that $\mu_{l}=0.113826, \sigma_{l}=0.002990$, and $\left.\lambda_{0}=0.015030\right)$.

- scenario 3: A pool size of $n=1000$, the parameter set from Table 3 except for $\mu_{l}=0.114375, \sigma_{l}=0.003179$, and $\lambda_{0}=0.015030$.

Scenario 1 has the highest unsystematic risk while scenario 3 has the highest systematic risk (as can be seen by the higher volatility $\sigma_{l}$ of the mortality rate). The parameters are chosen such that the overall mortality risk, that is, the variance $\operatorname{Var}_{\mathcal{F}_{0}}^{\mathbb{P}^{a} \times \mathbb{P}^{f}}\left(N_{T} / n\right)$, is the same in the three scenarios. Table 4 presents the insurance premiums for the different hybrid valuation operators. We observe that the two-step method and the conditional standard deviation principle lead to the same premium in all three scenarios. The three-step method, however, is able to treat unsystematic and systematic mortality risks 
differently. We observe that scenario 1 has the highest unsystematic risk premium $\pi_{0}^{a}\left(H_{T}^{(2)}\right)$ but the lowest systematic risk premium $\pi_{0}^{n}\left(H_{T}^{(3)}\right)$. Whether or not the premium in the three-step method increases with a higher share of systematic mortality risk, depends on the choice of the risk charge parameters $\alpha$ and $\theta^{(1)}$.

\section{DisCUSSION OF ASSUMPTIONS}

To focus on our main messages and to make the paper easily accessible, we have included several simplifying assumptions in our analysis. In this section, we want to point out some of them and discuss their effect on our results.

- Stochastic interest rates: It is generally accepted that a constant risk-free interest rate $r$ does not reflect empirical observations. We can assume that our financial market contains a time- $T$ stochastic discount factor $D_{T}:=$ $e^{-\int_{0}^{T} r_{s} \mathrm{~d} s}$, independent of the product's mortality risk. In such a setting, all our results are still valid (as long as the financial market remains complete, else see below). What we need to do is replace any financial claim $h_{j}\left(S_{T}\right)$ by a new financial claim $D_{T} \cdot h_{j}\left(S_{T}\right)$ (and set the (deterministic) discount rate to $r=0)$.

- Incomplete financial markets: As already mentioned in the introduction, financial markets are incomplete and financial claims cannot necessarily be completely hedged on financial markets. The focus of this article is on a complete financial market. It would nevertheless be desirable to extend the results in this article to incomplete financial markets. The general idea of decomposing the product's payoff in a hedgeable, a diversifiable, and a residual part is still applicable. However, a valuation operator in incomplete financial markets still faces at least two additional difficulties: first, the concept of market consistency in incomplete financial markets requires some discussion (see, e.g., Hirbod and Gospodinov, 2018). Second, a criterion is necessary (e.g., a specific hedging strategy) to separate between hedgeable financial risk (part $H_{T}^{(1)}$ ) and residual risk (part $H_{T}^{(3)}$ ). Such a separation has to be model-dependent, that is, the premium and payoff decomposition will depend on the hedging strategy chosen.

- Dependence between actuarial and financial risks: If financial and actuarial risks are dependent, the diversifiable part $H_{T}^{(2)}$ in decomposition (3.1) is still diversifiable. Applying the conditional law of large numbers, we obtain a similar result as in Theorem 3.1(a) for dependent risks, that is,

$$
\frac{\sum_{j=1}^{n} g_{j}\left(\tau_{j}\right)}{n} \mid \mathcal{G}_{T} \vee \mathcal{F}_{T}^{f} \stackrel{n \rightarrow \infty}{\longrightarrow} \mathbb{E}_{\mathcal{F}_{0}}^{\mathbb{P}^{a} \times \mathbb{P}^{f}}\left[g_{1}\left(\tau_{1}\right) \mid \mathcal{G}_{T} \vee \mathcal{F}_{T}^{f}\right] \quad \text { a.s. }
$$


For dependent financial and actuarial risks, we can decompose the mortality-risk-related part of the payoff in four different parts:

$$
\begin{aligned}
g_{j}\left(\tau_{j}\right)= & \underbrace{\mathbb{E}_{\mathcal{F}_{0}}^{\mathbb{P}^{a} \times \mathbb{P}^{f}}\left[g_{j}\left(\tau_{j}\right)\right]}_{\text {best-estimate }}+\underbrace{\left(g_{j}\left(\tau_{j}\right)-\mathbb{E}_{\mathcal{F}_{0} \vee \mathcal{G}_{T} \vee \mathcal{F}_{T}^{f}}^{\mathbb{P}^{a} \times \mathbb{P}^{f}}\left[g_{j}\left(\tau_{j}\right)\right]\right)}_{\text {unsystematic deviation }} \\
& +\underbrace{\left(\mathbb{E}_{\mathcal{F}_{0} \vee \mathcal{G}_{T} \vee \mathcal{F}_{T}^{f}}^{\mathbb{P}^{a} \times \mathbb{P}^{f}}\left[g_{j}\left(\tau_{j}\right)\right]-\mathbb{E}_{\mathcal{F}_{0} \vee \mathcal{F}_{T}^{f}}^{\mathbb{P}^{a} \times \mathbb{P}^{f}}\left[g_{j}\left(\tau_{j}\right)\right]\right)}_{\text {systematic deviation, systematic mortality part }} \\
& +\underbrace{\left(\mathbb{E}_{\mathcal{F}_{0} \vee \mathcal{F}_{T}^{f}}^{\mathbb{P}^{a} \times \mathbb{P}^{f}}\left[g_{j}\left(\tau_{j}\right)\right]-\mathbb{E}_{\mathcal{F}_{0}}^{\mathbb{P}^{a} \times \mathbb{P}^{f}}\left[g_{j}\left(\tau_{j}\right)\right]\right)}_{\text {systematic deviation, financial part }} .
\end{aligned}
$$

This can again be used to define a premium decomposition (3.1). The first term in (8.1) relates to the financial, hedgeable part $H_{T}^{(1)}$, namely:

$$
H_{T}^{(1)}:=\frac{1}{n} \sum_{j=1}^{n} \mathbb{E}_{\mathcal{F}_{0}}^{\mathbb{P}^{a} \times \mathbb{P}^{f}}\left[g_{j}\left(\tau_{j}\right)\right] \cdot h_{j}\left(S_{T}\right) .
$$

If financial and systematic mortality risks were independent, we obtain $\mathbb{E}_{\mathcal{F}_{0} \vee \mathcal{F}_{T}^{f}}^{\mathbb{P}^{a} \times \mathbb{P}^{f}}\left[g_{j}\left(\tau_{j}\right)\right]=\mathbb{E}_{\mathcal{F}_{0}}^{\mathbb{P}^{a} \times \mathbb{P}^{f}}\left[g_{j}\left(\tau_{j}\right)\right]$ and (8.1) reduces to the premium decomposition (3.1). Stated differently, our previous results can be seen as a special case of the decomposition (8.1). The explanation for the additional (last) $\mathcal{F}_{T}^{f}$-measurable term in (8.1) is the fact that the dependence between financial and actuarial risks allows to partially hedge systematic mortality risk on financial markets. The second part relates to the diversifiable, actuarial part:

$$
H_{T}^{(2)}:=\frac{1}{n} \sum_{j=1}^{n}\left(g_{j}\left(\tau_{j}\right)-\mathbb{E}_{\mathcal{F}_{0} \vee \mathcal{G}_{T} \vee \mathcal{F}_{T}^{f}}^{\mathbb{P}^{a} \times \mathbb{P}^{f}}\left[g_{j}\left(\tau_{j}\right)\right]\right) \cdot h_{j}\left(S_{T}\right) .
$$

The remaining two terms can be used to define the residual part that is neither hedgeable nor diversifiable and consists of a combination of systematic mortality risk and financial risk:

$$
H_{T}^{(3)}:=\frac{1}{n} \sum_{j=1}^{n}\left(\mathbb{E}_{\mathcal{F}_{0} \vee \mathcal{G}_{T} \vee \mathcal{F}_{T}^{f}}^{\mathbb{P}^{a} \times \mathbb{P}^{f}}\left[g_{j}\left(\tau_{j}\right)\right]-\mathbb{E}_{\mathcal{F}_{0}}^{\mathbb{P}^{a} \times \mathbb{P}^{f}}\left[g_{j}\left(\tau_{j}\right)\right]\right) \cdot h_{j}\left(S_{T}\right) .
$$

Overall, the case of dependent financial and actuarial risks allows us to also define a premium decomposition similar to (3.1). 


\section{CONCLUSION}

We have introduced a novel hybrid valuation operator based on a decomposition of the product's payoff in a financial, hedgeable part, a diversifiable part, and a neither hedgeable nor diversifiable part. The risk of the first part can be reduced by hedging on financial markets, while the second part can be removed by diversifying in a sufficiently large portfolio. To valuate also the residual risk that cannot be hedged nor diversified, we have suggested to use the Esscher transform to choose a suitable martingale measure. We have demonstrated that this valuation operator satisfies the desirable properties of a valuation operator and generates a feasible underlying strategy of risk management. It is one possibility to price hybrid financial and actuarial products "interpolating" between risk-neutral pricing in finance and actuarial valuation in insurance. The threestep method allows to separately treat diversifiable and systematic mortality risk and suggest strategies to manage the risks.

In this work, we restrict ourselves to a static valuation, postulating that, typically, life insurance products are not liquidly traded on exchanges. It would nevertheless be desirable to extend this analysis to the continuous-time case to compute, for example, the product's actuarial reserves at intermediate time points. In this case, it is important to also discuss issues like time consistency of this valuation operator (see also, e.g., Pelsser and Stadje, 2014; Pelsser and Ghalehjooghi, 2016).

\section{ACKNOWLEDGMENTS}

The authors would like to thank the anonymous referees for many useful comments that helped to improve earlier versions of this manuscript. They further want to thank participants of the European Actuarial Conference in Leuven 2018, AFMathConf 2019, the workshop "Fair valuation in Insurance" in Brussels and the 23rd International Congress on Insurance: Mathematics and Economics in Munich 2019 for fruitful discussions that helped to improve earlier versions of this manuscript. The authors acknowledge support by the FNRS cross-university grant PDR "Risk management and Pricing in Finance and Insurance". The work leading to this publication was supported by the PRIME program of the German Academic Exchange Service (DAAD) with funds from the German Federal Ministry of Education and Research (BMBF).

\section{NOTES}

1. Technically, we assume that $\mathbb{E}_{\mathcal{F}_{0}}^{\mathbb{P}^{a} \times \mathbb{Q}^{f}}\left[g_{j}\left(\tau_{j}\right) \cdot h_{j}\left(S_{T}\right)\right]$ and $\mathbb{E}_{\mathcal{F}_{0}}^{\mathbb{P}^{a} \times \mathbb{P}^{f}}\left[g_{j}\left(\tau_{j}\right) \cdot h_{j}\left(S_{T}\right)\right]$ are also finite for each $j=1,2, \ldots, n$. Further, the variances $\operatorname{Var}_{\mathcal{F}_{0}}^{\mathbb{P}^{a} \times \mathbb{Q}^{f}}\left(g_{j}\left(\tau_{j}\right) \cdot h_{j}\left(S_{T}\right)\right)$ and $\operatorname{Var}_{\mathcal{F}_{0}}^{\mathbb{P}^{a} \times \mathbb{P}^{f}}\left(g_{j}\left(\tau_{j}\right) \cdot h_{j}\left(S_{T}\right)\right)$ are assumed to be finite for each $j=1,2, \ldots, n$.

2. If $\boldsymbol{X}_{T}$ is normally distributed with mean vector $\boldsymbol{\mu}:=\left(\mu^{(1)}, \mu^{(2)}, \ldots, \mu^{(k)}\right)^{\prime}$ and covariance matrix $\boldsymbol{\Sigma}$ with elements $\Sigma_{i, j}:=\operatorname{Cov}\left(X_{T}^{(i)}, X_{T}^{(j)}\right)$ for $i, j=1,2, \ldots, n$ under a probability measure $\tilde{P}$, then, the moment generating function of $\boldsymbol{X}_{T}$ under $\tilde{P}$ is, for $\boldsymbol{u} \in \mathbb{R}^{n \times 1}$, given by 


$$
\boldsymbol{M}(\boldsymbol{u}, T):=\mathbb{E}_{\mathcal{F}_{0}}^{\tilde{P}}\left[e^{\left\langle\boldsymbol{u}, \boldsymbol{X}_{T}\right\rangle}\right]=\exp \left(\boldsymbol{u}^{\prime} \boldsymbol{\mu}+\frac{1}{2} \boldsymbol{u}^{\prime} \boldsymbol{\Sigma} \boldsymbol{u}\right)
$$

For $\boldsymbol{\theta}:=\left(\theta^{(1)}, \theta^{(2)}, \ldots, \theta^{(k)}\right)^{\prime} \in \mathbb{R}^{k \times 1}$, we can conclude that (see, e.g., Gerber and Shiu, 1994, p. $119 \mathrm{ff}$ and p. 126ff)

$$
\boldsymbol{M}(\boldsymbol{u}, T ; \boldsymbol{\theta}):=\frac{\mathbb{E}_{\mathcal{F}_{0}}^{\tilde{P}}\left[e^{\left(\boldsymbol{u}-\boldsymbol{\theta}, \boldsymbol{X}_{T}\right\rangle}\right]}{\mathbb{E}_{\mathcal{F}_{0}}^{\tilde{P}}\left[e^{\left(-\boldsymbol{\theta}, \boldsymbol{X}_{T}\right\rangle}\right]}=\frac{\boldsymbol{M}(\boldsymbol{u}-\boldsymbol{\theta}, T)}{\boldsymbol{M}(-\boldsymbol{\theta}, T)}=\exp \left(\boldsymbol{u}^{\prime}(\boldsymbol{\mu}-\boldsymbol{\Sigma} \cdot \boldsymbol{\theta})+\frac{1}{2} \boldsymbol{u}^{\prime} \boldsymbol{\Sigma} \boldsymbol{u}\right),
$$

that is, the Esscher transform with parameter vector $\boldsymbol{\theta}$ leads again to an $k$-variate normal distribution, where the mean vector is modified from $\boldsymbol{\mu}$ to $\boldsymbol{\mu}-\boldsymbol{\Sigma} \cdot \boldsymbol{\theta}$.

For normally distributed risks, the Esscher transform leads to the same transformation as the Wang transform (see also Kijima, 2006). These observations also follow from more general results in, for example, Theorem 4.1 of Eberlein et al. (2009), see also Ballotta et al. (2017).

3. Note that we do not consider monotonicity, that is, if $G_{T}^{n}<H_{T}^{n}$ for two claims $G_{T}^{n}, H_{T}^{n} \in \mathcal{H}_{T}$, then $\pi_{0}\left(G_{T}^{n}\right) \leq \pi_{0}\left(H_{T}^{n}\right)$, and this although one can classify this as a relevant property for valuation operators. The reason hereto is that this property is in general not satisfied for many common actuarial principles, for example, the standard deviation principle (see, e.g., Kaas et al., 2008 for examples and a more detailed discussion).

4. Annual survival probabilities of an $x$-year-old female are given by:

$$
{ }_{1} \hat{p}_{x}=s \cdot g^{c^{x}(c-1)}, \quad \text { where } 0<s \leq 1, \quad 0<g<1, \quad \text { and } \quad c>1 .
$$

For women, we choose the survival specification $(\mathbf{F R})$ where the Royal Decree parameters are

$$
s=0.999669730966, \quad g=0.999951440172, \quad \text { and } \quad c=1.116792453830 .
$$

5. See http://www.mortality.org.

6. Similarly, for the two-step method and the conditional standard deviation principle in Section 4.2 , we use that

$$
\begin{aligned}
\operatorname{Var}_{\mathcal{F}_{0}}^{\mathbb{P}^{a} \times \mathbb{P}^{f}}\left(\frac{1}{n} \sum_{j=1}^{n} g\left(\tau_{j}\right)\right) & =\frac{1}{n^{2}} \operatorname{Var}_{\mathcal{F}_{0}}^{\mathbb{P}^{p} \times \mathbb{P}^{f}}\left(N_{T}\right) \\
& =\frac{1}{n^{2}}\left(\operatorname{Var}_{\mathcal{F}_{0}}^{\mathbb{p}^{p a} \times \mathbb{P}^{f}}\left(\mathbb{E}_{\mathcal{G}_{T} \vee \mathcal{F}_{0}}^{\mathbb{F}^{a} \times \mathbb{P}^{f}}\left[N_{T}\right]\right)+\mathbb{E}_{\mathcal{F}_{0}}^{\mathbb{P} \times \mathbb{P}^{f}}\left[\operatorname{Var}_{\mathcal{G}_{T} \vee \mathcal{F}_{0}}^{\mathbb{P}^{a} \mathbb{P}^{f}}\left(N_{T}\right)\right]\right) \\
& =\operatorname{Var}_{\mathcal{F}_{0}}^{\mathbb{p}^{a} \times \mathbb{P}^{f}}\left(e^{-\int_{0}^{T} \lambda_{s} \mathrm{~d} s}\right)+\frac{1}{n} \mathbb{E}_{\mathcal{F}_{0}}^{\mathbb{P}^{a} \times \mathbb{P}^{f}}\left[e^{-\int_{0}^{T} \lambda_{s} \mathrm{~d} s}\left(1-e^{-\int_{0}^{T} \lambda_{s} \mathrm{~d} s}\right)\right] .
\end{aligned}
$$

For the mortality model introduced in Example 5.3, we find that:

$$
\begin{aligned}
\left.\mathbb{E}_{\mathcal{F}_{0}}^{\mathbb{P}^{a} \times \mathbb{P}^{f}}\left[e^{-\int_{0}^{T} \lambda_{s} \mathrm{~d} s}\left(1-e^{-\int_{0}^{T} \lambda_{s} \mathrm{~d} s}\right)\right)\right] & =e^{-m_{0, T}+\frac{1}{2} s_{0, T}^{2}}-e^{-2 m_{0, T}+2 s_{0, T}^{2}}, \\
\operatorname{Var}_{\mathcal{F}_{0}}^{\mathbb{P}^{a} \times \mathbb{P}^{f}}\left(e^{-\int_{0}^{T} \lambda_{s} \mathrm{~d} s}\right) & =\left(e^{s_{0, T}^{2}}-1\right) \cdot e^{-2 m_{0, T}+s_{0, T}^{2}}
\end{aligned}
$$

\section{REFERENCES}

Ballotta, L., Deelstra, G. and Rayée, G. (2017) Multivariate FX models with jumps: Triangles, quantos and implied correlation. European Journal of Operational Research, 260(3), $1181-1199$.

BARIgOU, K. and DHAENE, J. (2019) Fair valuation of insurance liabilities via mean-variance hedging in a multi-period setting. Scandinavian Actuarial Journal, 2, 163-187.

Bielecki, T.R. and RutKowski, M. (2004) Credit Risk: Modeling, Valuation and Hedging. Berlin, Heidelberg, Springer. 
BOYLE, P.P. and SCHWARTZ, E.S. (1977) Equilibrium prices of guarantees under equity-linked contracts. Journal of Risk and Insurance, 44(4), 639-660.

BRENNAN, M.J. and SCHWARTZ, E.S. (1979) Alternative investment strategies for the issuers of equity-linked life insurance with an asset value guarantee. Journal of Business, 52(1), 63-93.

Chen, A., Hieber, P. and Klein, J.K. (2019) Tonuity: A novel individual-oriented retirement plan. ASTIN Bulletin, 49(1), 5-30.

Chen, A. and Vigna, E. (2017) A unisex stochastic mortality model to comply with EU gender directive. Insurance: Mathematics \& Economics, 73, 124-136.

Cont, R. and Tankov, P. (2003) Financial Modelling with Jump Processes. Boca Raton, London, New York, Chapman \& Hall/CRC Financial Mathematics Series.

DAHL, M. and MøLler, T. (2006) Valuation and hedging of life insurance liabilities with systematic mortality risk. Insurance: Mathematics and Economics, 39(2), 193-217.

Delong, Ł., Dhaene, J. and BARIGOU, K. (2019a) Fair valuation of insurance liability cashflow streams in continuous time: Applications. ASTIN Bulletin, 49(2), 299-333.

Delong, Ł., Dhaene, J. and BARIGOU, K. (2019b) Fair valuation of insurance liability cashflow streams in continuous time: Theory. Insurance: Mathematics \& Economics, 88, 196-208.

Dhaene, J., Stassen, B., Barigou, K., Linders, D. and Chen, Z. (2017) Fair valuation of insurance liabilities: Merging actuarial judgement and market-consistency. Insurance: Mathematics and Economics, 76, 14-27.

Eberlein, E., Papapantoleon, A. and Shiryaev, A. (2009) Esscher transform and the duality principle for multidimensional semimartingales. The Annals of Applied Probability, 19, 1944-1971.

Engsner, H., Lindensjö, K. and Lindskog, F. (2020) The value of a liability cash flow in discrete time subject to capital requirements. Finance and Stochastics, 24(1), 125-167.

GER BER, H.U. (1997) Life Insurance Mathematics, 3rd edition. Springer.

GERBER, H.U. and SHIU, E.S.W. (1994) Option pricing by Esscher transform. Transactions of Society of Actuaries, 46, 99-191.

Gerber, H.U. and ShiU, E.S.W. (1996) Actuarial bridges to dynamic hedging and option pricing. Insurance: Mathematics and Economics, 18(3), 183-218.

HiR BOD, A. and GospodinOv, N. (2018) Market consistent valuation with financial imperfection. Decision in Economics and Finance, 41, 65-90.

Ikeda, N. and Watanabe, S. (2014) Stochastic Differential Equations and Diffusion Processes. Elsevier.

KaAs, R., Goovaerts, M., Dhaene, J. and Denuit, M. (2008) Modern Actuarial Risk Theory: Using R, Vol. 128. Springer Science \& Business Media.

Keller, P. and Luder, T. (2004) White paper of the Swiss Solvency Test. Swiss Federal Office of Private Insurance, Switzerland.

KiJIMA, M. (2006) A multivariate extension of equilibrium pricing transform: The multivariate Esscher and Wang transform for pricing financial and insurance risks. ASTIN Bulletin, 36(1), 269-283.

Laeven, R.J. and Goovaerts, M.J. (2008) Premium calculation and insurance pricing. In Encyclopedia of Quantitative Risk Analysis and Assessment, vol. 3, pp. 1302-1314.

LIN, Y. and CoX, S. (2008) Securitization of catastrophe mortality risks. Insurance: Mathematics \& Economics, 42(2), 628-637.

Luciano, E., Regis, L. and Vigna, E. (2012) Delta-gamma hedging of mortality and interest rate risk. Insurance: Mathematics and Economics, 50(3), 402-412.

LuCiano, E. and VignA, E. (2008) Mortality risk via affine stochastic intensities: Calibration and empirical relevance. Belgian Actuarial Journal, 8(1), 5-16.

Malamud, S., Trubowitz, E. and WÜthrich, M.V. (2008) Market consistent pricing of insurance products. Astin Bulletin, 38(2), 483-526.

MöHR, C. (2011) Market-consistent valuation of insurance liabilities by cost of capital. ASTIN Bulletin, 41(2), 315-341.

MøLLER, T. (2002) On valuation and risk management at the interface of insurance and finance. British Actuarial Journal, 8(4), 787-827.

Pelsser, A. and Ghalehjooghi, A.S. (2016) Time-consistent actuarial valuations. Insurance: Mathematics \& Economics, 66, 97-112. 
Pelsser, A. and Stadje, M. (2014) Time-consistent and market-consistent evaluations. Mathematical Finance, 24(1), 25-65.

Perlman, M.D. (1974) Jensen's inequality for a convex vector-valued function on an infinitedimensional space. Journal of Multivariate Analysis, 4, 52-65.

Rotar, V.I. (2014) Actuarial Models: the Mathematics of Insurance. Boca Raton, London, New York, CRC Press.

Schoutens, W. (2003) Lévy Processes in Finance: Pricing Financial Derivatives. Berlin, Heidelberg, John Wiley \& Sons, Ltd.

TsAi, J. and TZENG, L. (2013) Securitization of catastrophe mortality risks. Astin Bulletin, 43(2), $97-121$.

Zeddouk, F. and Devolder, P. (2019) Pricing of longevity derivatives and cost of capital. Risks, 7(2), 1-29.

GRISELDA DEELSTRA

Department of Mathematics

Université libre de Bruxelles

Boulevard du Triomphe, 1050 Brussels, Belgium

PierRe DeVolder

Institute of Statistics, Biostatistics and Actuarial Sciences (ISBA/LIDAM)

Université Catholique de Louvain

20 Voie du Roman Pays, 1348 Louvain la Neuve, Belgium

KOSSI GNAMEHO

Department of Mathematics

Université libre de Bruxelles

Boulevard du Triomphe, 1050 Brussels, Belgium

PETER HIEBER (Corresponding author)

Institute of Statistics, Biostatistics and Actuarial Sciences (ISBA/LIDAM)

Université Catholique de Louvain

20 Voie du Roman Pays, 1348 Louvain la Neuve, Belgium

and

Institute of Insurance Science

University of Ulm

Helmholtzstr. 20, 89069 Ulm, Germany

E-Mail:peter.hieber@uni-ulm.de

\section{APPENDIX A}

\section{A.1. Proof of Theorem 3.1}

(a) Knowing that $\mathbb{E}_{\mathcal{F}_{0}}^{\mathbb{P}^{a} \times \mathbb{P}^{f}}\left[h_{j}\left(S_{T}\right)\right]<\infty$ and applying the tower rule of conditional expectation, we find that

$$
\mathbb{E}_{\mathcal{F}_{0}}^{\mathbb{P}^{a} \times \mathbb{P}^{f}}\left[H_{T}^{(2)}\right]=\mathbb{E}_{\mathcal{F}_{0}}^{\mathbb{P}^{a} \times \mathbb{P}^{f}}\left[\frac{1}{n} \sum_{j=1}^{n}\left(g_{j}\left(\tau_{j}\right)-\mathbb{E}_{\mathcal{F}_{0} \vee \mathcal{G}_{T}}^{\mathbb{P}^{a} \times \mathbb{P}^{f}}\left[g_{j}\left(\tau_{j}\right)\right]\right) \cdot h_{j}\left(S_{T}\right)\right]=0 .
$$


As $\operatorname{Var}_{\mathcal{F}_{0}}^{\mathbb{P}^{a} \times \mathbb{P}^{f}}\left(g_{j}\left(\tau_{j}\right)\right)<\infty$ and $\operatorname{Var}_{\mathcal{F}_{0}}^{\mathbb{P}^{a} \times \mathbb{P}^{f}}\left(h_{j}\left(S_{T}\right)\right)<\infty$, the payoff $H_{T}^{(2)}$ has finite variation. Knowing that the death times $\tau_{j}$ are independent given the systematic mortality information $\mathcal{G}_{T}$, we can apply the conditional law of large numbers to obtain

$$
\frac{\sum_{j=1}^{n} g_{j}\left(\tau_{j}\right)}{n} \mid \mathcal{G}_{T} \stackrel{n \rightarrow \infty}{\longrightarrow} \mathbb{E}_{\mathcal{F}_{0}}^{\mathbb{P}^{a} \times \mathbb{P}^{f}}\left[g_{1}\left(\tau_{1}\right) \mid \mathcal{G}_{T}\right] \quad \text { a.s. }
$$

Now, we can show that for each path of financial risk and systematic mortality risk (represented by the filtration $\mathcal{F}^{f} \vee \mathcal{G}$ ), the term $H_{T}^{(2)}$ disappears for $n \rightarrow \infty$ :

$$
\begin{aligned}
H_{T}^{(2)} \mid \mathcal{F}_{T}^{f} \vee \mathcal{G}_{T} & =\frac{\sum_{j=1}^{n}\left(g_{j}\left(\tau_{j}\right)-\mathbb{E}_{\mathcal{F}_{0} \vee \mathcal{G}_{T}}^{\mathbb{P}^{a} \times \mathbb{P}^{f}}\left[g_{j}\left(\tau_{j}\right)\right]\right) \cdot h_{j}\left(S_{T}\right)}{n} \mid \mathcal{F}_{T}^{f} \vee \mathcal{G}_{T} \\
& \stackrel{n \rightarrow \infty}{\longrightarrow} \mathbb{E}_{\mathcal{F}_{0}}^{\mathbb{P}^{a} \times \mathbb{P}^{f}}\left[\left(g_{1}\left(\tau_{1}\right)-\mathbb{E}_{\mathcal{F}_{0} \vee \mathcal{G}_{T}}^{\mathbb{P}^{a} \times \mathbb{P}^{f}}\left[g_{1}\left(\tau_{1}\right)\right]\right) \cdot h_{1}\left(S_{T}\right) \mid \mathcal{F}_{T}^{f} \vee \mathcal{G}_{T}\right]=0 \text { a.s. }
\end{aligned}
$$

where we exploit the conditional independence of individual deaths and the independence between unsystematic mortality risk (filtration $\vee_{j=1}^{n} \mathcal{I}^{j}$ ) and systematic mortality/financial risks (filtration $\mathcal{F}^{f} \vee \mathcal{G}$ ).

(b) From (5.5), we obtain in case of deterministic mortality rates that $\mathbb{E}_{\mathcal{F}_{0} \vee \mathcal{G}_{T}}^{\mathbb{P}^{a} \times \mathbb{P}^{f}}\left[g_{j}\left(\tau_{j}\right)\right]=$ $\mathbb{E}_{\mathcal{F}_{0}}^{\mathbb{P}^{\mathrm{a}} \mathbb{P}^{f}}\left[g_{j}\left(\tau_{j}\right)\right]$ for all $j=1,2, \ldots, n$. This immediately yields the desired claim.

\section{APPENDIX B}

\section{B.1. Proof of Theorem 4.2}

If $\pi_{0}^{a}, \pi_{0}^{f}$ and $\pi_{0}^{n}$ are valuation operators in the sense of Definition 4.1, it is straightforward to show that Definition 4.1 (a)-(d) also holds for (4.3), (4.4), and (4.1).

Sub-additivity (e) for the two-step premium (4.3) follows from the sub-additivity of $\pi_{0}^{a}$ and $\pi_{0}^{f}$ :

$$
\begin{aligned}
\pi_{0}^{(2 a)}\left(G_{T}^{n}+H_{T}^{n}\right) & :=\pi_{0}^{f}\left(e^{r T} \pi_{0}^{a}\left(G_{T}^{n}+H_{T}^{n} \mid \mathcal{F}_{T}^{f} \vee \mathcal{F}_{0}\right) \mid \mathcal{F}_{0}\right) \\
& \leq \pi_{0}^{f}\left(e^{r T} \pi_{0}^{a}\left(G_{T}^{n} \mid \mathcal{F}_{T}^{f} \vee \mathcal{F}_{0}\right)+e^{r T} \pi_{0}^{a}\left(H_{T}^{n} \mid \mathcal{F}_{T}^{f} \vee \mathcal{F}_{0}\right) \mid \mathcal{F}_{0}\right) \\
& \leq \pi_{0}^{f}\left(e^{r T} \pi_{0}^{a}\left(G_{T}^{n} \mid \mathcal{F}_{T}^{f} \vee \mathcal{F}_{0}\right) \mid \mathcal{F}_{0}\right)+\pi_{0}^{f}\left(e^{r T} \pi_{0}^{a}\left(H_{T}^{n} \mid \mathcal{F}_{T}^{f} \vee \mathcal{F}_{0}\right) \mid \mathcal{F}_{0}\right) \\
& =\pi_{0}^{(2 a)}\left(G_{T}^{n}\right)+\pi_{0}^{(2 a)}\left(H_{T}^{n}\right) .
\end{aligned}
$$

Similarly, the sub-additivity of $\pi_{0}^{a}, \pi_{0}^{f}$, and $\pi_{0}^{n}$ directly implies the sub-additivity of the threestep method.

To show that the conditional standard deviation principle (4.4) is sub-additive, we use a two-dimensional version of Jensen's inequality (see, e.g., Perlman, 1974): for the concave function $f(x, y)=\sqrt{x \cdot y}$, we find that $\mathbb{E}[f(X, Y)] \leq f(\mathbb{E}[X], \mathbb{E}[Y])$. Applying this for $X:=$ $\operatorname{Var}_{\mathcal{F}_{T}^{f} \vee \mathcal{F}_{0}}^{\mathbb{P}^{a}}\left(G_{T}^{n}\right)$ and $Y:=\operatorname{Var}_{\mathcal{F}_{T}^{f} \mathbb{P}^{a}}^{\mathbb{P}^{f}}\left(H_{T}^{n}\right)$, we obtain 


$$
\begin{aligned}
& \mathbb{E}_{\mathcal{F}_{0}}^{\mathbb{P}^{a} \times \mathbb{P}^{f}}\left[\operatorname{Var}_{\mathcal{F}_{T}^{f} \vee \mathcal{F}_{0}}^{\mathbb{P}^{a} \times \mathbb{P}^{f}}\left(G_{T}^{n}+H_{T}^{n}\right)\right] \\
& \leq \mathbb{E}_{\mathcal{F}_{0}}^{\mathbb{P}^{a} \times \mathbb{P}^{f}}\left[\operatorname{Var}_{\mathcal{F}_{T}^{f} \vee \mathcal{F}_{0}}^{\mathbb{P}^{a} \times \mathbb{P} f}\left(G_{T}^{n}\right)+\operatorname{Var}_{\mathcal{F}_{T}^{f} \vee \mathcal{F}_{0}}^{\mathbb{P}^{a} \times \mathbb{P}^{f}}\left(H_{T}^{n}\right)+2 \sqrt{\operatorname{Var}_{\mathcal{F}_{T}^{f} \mathbb{P}^{a} \times \mathcal{F}_{0} f}^{f}\left(G_{T}^{n}\right) \cdot \operatorname{Var}_{\mathcal{F}_{T}^{f} \mathbb{P}^{a} \times \mathcal{F}_{0}}^{\mathbb{P}^{f}}\left(H_{T}^{n}\right)}\right] \\
& \leq \mathbb{E}_{\mathcal{F}_{0}}^{\mathbb{P}^{a} \times \mathbb{P}^{f}}\left[\operatorname{Var}_{\mathcal{F}_{T}^{f} \vee \mathcal{F}_{0}}^{\mathbb{P}^{a} \times \mathbb{P}^{f}}\left(G_{T}^{n}\right)+\operatorname{Var}_{\mathcal{F}_{T}^{f} \vee \mathcal{F}_{0}}^{\mathbb{P}^{a} \times \mathbb{P}^{f}}\left(H_{T}^{n}\right)\right] \\
& +2 \sqrt{\mathbb{E}_{\mathcal{F}_{0}}^{\mathbb{P a} \times \mathbb{P}^{f}}\left[\operatorname{Var}_{\mathcal{F}_{T}^{f} \mathbb{P}^{a} \times \mathbb{F}_{0}^{f f}}^{f}\left(G_{T}^{n}\right)\right]} \cdot \sqrt{\mathbb{E}_{\mathcal{F}_{0}}^{\mathbb{P a} \times \mathbb{P}^{f}}\left[\operatorname{Var}_{\mathcal{F}_{T}^{f} \vee \mathcal{F}_{0}}^{\mathbb{P}^{a} \times \mathbb{P}^{f}}\left(H_{T}^{n}\right)\right]} \\
& =\left(\sqrt{\mathbb{E}_{\mathcal{F}_{0}}^{\mathbb{P}^{a} \times \mathbb{P}^{f}}\left[\operatorname{Var}_{\mathcal{F}_{T}^{f} \vee \mathcal{F}_{0}}^{\mathbb{P}^{a} \times \mathbb{P}^{f}}\left(G_{T}^{n}\right)\right]}+\sqrt{\mathbb{E}_{\mathcal{F}_{0}}^{\mathbb{P}^{a} \times \mathbb{P}^{f}}\left[\operatorname{Var}_{\mathcal{F}_{T}^{f}{ }^{\mathbb{P}^{a}} \times \mathbb{F}_{0}^{f}}^{f}\left(H_{T}^{n}\right)\right]}\right)^{2}
\end{aligned}
$$

to conclude that

$$
\begin{aligned}
\pi_{0}^{(2 b)}\left(G_{T}^{n}+H_{T}^{n}\right) & :=e^{-r T}\left(\mathbb{E}_{\mathcal{F}_{0}}^{\mathbb{P}^{a} \times \mathbb{Q}^{f}}\left[G_{T}^{n}+H_{T}^{n}\right]+\frac{\alpha}{2} \sqrt{\mathbb{E}_{\mathcal{F}_{0}}^{\mathbb{P}^{a} \times \mathbb{P}^{f}}\left[{\operatorname{\operatorname {Var}^{P}}}_{\mathcal{F}_{T}^{f} \mathbb{P}^{a} \times \mathcal{P}_{0}}^{f}\left(G_{T}^{n}+H_{T}^{n}\right)\right]}\right. \\
& \leq \pi_{0}^{(2 b)}\left(G_{T}^{n}\right)+\pi_{0}^{(2 b)}\left(H_{T}^{n}\right) .
\end{aligned}
$$

For a financial, hedgeable payoff $h_{1}\left(S_{T}\right) \in \mathcal{H}_{T}^{f}$, the decomposition (3.1) contains only the first part $H_{T}^{(1)}$. Then, by the linearity of expectation, we find that the three-step valuation operator is market-consistent, that is, for any claim $H_{T}^{n} \in \mathcal{H}_{T}$ it holds that

$$
\pi_{0}^{(3)}\left(H_{T}^{n}+h_{1}\left(S_{T}\right)\right)=\pi_{0}^{(3)}\left(H_{T}^{n}\right)+\pi_{0}^{f}\left(h_{1}\left(S_{T}\right) \mid \mathcal{F}_{0}\right) \text {. }
$$

For the two-step method, we can apply translation invariance to obtain

$$
\begin{aligned}
\pi_{0}^{(2 a)}\left(H_{T}^{n}+h_{1}\left(S_{T}\right)\right) & :=\pi_{0}^{f}\left(e^{r T} \pi_{0}^{a}\left(H_{T}^{n}+h_{1}\left(S_{T}\right) \mid \mathcal{F}_{T}^{f} \vee \mathcal{F}_{0}\right) \mid \mathcal{F}_{0}\right) \\
& =\pi_{0}^{(2 a)}\left(H_{T}^{n}\right)+\pi_{0}^{f}\left(h_{1}\left(S_{T}\right) \mid \mathcal{F}_{0}\right)
\end{aligned}
$$

For the conditional standard deviation principle (4.4), we find that:

$$
\begin{aligned}
\pi_{0}^{(2 b)} & \left(H_{T}^{n}+h_{1}\left(S_{T}\right)\right) \\
& :=e^{-r T}\left(\mathbb{E}_{\mathcal{F}_{0}}^{\mathbb{P}^{a} \times \mathbb{Q}^{f}}\left[H_{T}^{n}+h_{1}\left(S_{T}\right)\right]+\frac{\alpha}{2} \sqrt{\mathbb{E}_{\mathcal{F}_{0}}^{\mathbb{P a} \times \mathbb{P}^{f}}\left[\operatorname{Var}_{\mathcal{F}_{T}^{f} \times \mathcal{F}_{0}}^{\mathbb{P}^{a} \times \mathbb{P}^{f}}\left(H_{T}^{n}+h_{1}\left(S_{T}\right)\right)\right]}\right) \\
& =e^{-r T}\left(\mathbb{E}_{\mathcal{F}_{0}}^{\mathbb{P}^{a} \times \mathbb{Q}^{f}}\left[H_{T}^{n}\right]+\frac{\alpha}{2} \sqrt{\mathbb{E}_{\mathcal{F}_{0}}^{\mathbb{P}^{a} \times \mathbb{P} f}\left[\operatorname{Var}_{\mathcal{F}_{T}^{f} \vee \mathcal{F}_{0}}^{\mathbb{P}^{a} \times \mathbb{P}^{f}}\left(H_{T}^{n}\right)\right]}\right)+\pi_{0}^{f}\left(h_{1}\left(S_{T}\right) \mid \mathcal{F}_{0}\right) \\
& =\pi_{0}^{(2 b)}\left(H_{T}^{n}\right)+\pi_{0}^{f}\left(h_{1}\left(S_{T}\right) \mid \mathcal{F}_{0}\right) .
\end{aligned}
$$

Next, we consider a purely actuarial claim $g_{T}^{n}:=\frac{1}{n} \sum_{j=1}^{n} g_{j}\left(\tau_{j}\right) \in \mathcal{H}_{T}^{a}$ that does not contain any systematic mortality risk (i.e., a portfolio of insureds whose remaining lifetimes $\tau_{j}, j=1,2, \ldots, n$, are independent and identically distributed (i.i.d.)). In this case, the 
decomposition (3.1) contains only $H_{T}^{(1)}$ and $H_{T}^{(2)}$. Then, (4.1) simplifies to

$$
\pi_{0}^{(3)}\left(g_{T}^{n}\right)=e^{-r T} \mathbb{E}_{\mathcal{F}_{0}}^{\mathbb{P}^{a} \times \mathbb{P}^{f}}\left[g_{T}^{n}\right]+\pi_{0}^{a}\left(g_{T}^{n}-\mathbb{E}_{\mathcal{F}_{0}}^{\mathbb{P}}\left[g_{T}^{n}\right] \mid \mathcal{F}_{0}\right)=\pi_{0}^{a}\left(g_{T}^{n} \mid \mathcal{F}_{0}\right)=\pi_{0}^{(2 a)}\left(g_{T}^{n}\right) .
$$

For the conditional standard deviation principle (4.4), we find that:

$$
\begin{aligned}
\pi_{0}^{(2 b)}\left(g_{T}^{n}\right) & :=e^{-r T}\left(\mathbb{E}_{\mathcal{F}_{0}}^{\mathbb{P}^{a} \times \mathbb{Q}^{f}}\left[g_{T}^{n}\right]+\frac{\alpha}{2} \sqrt{\mathbb{E}_{\mathcal{F}_{0}}^{\mathbb{P}^{a} \times \mathbb{P}^{f}}\left[\operatorname{Var}_{\mathcal{F}_{T}^{f} \mathbb{P}^{a} \times \mathbb{F}^{f}}^{\mathbb{P}^{f}}\left(g_{T}^{n}\right)\right]}\right) \\
& =e^{-r T}\left(\mathbb{E}_{\mathcal{F}_{0}}^{\mathbb{P}^{a} \times \mathbb{Q}^{f}}\left[g_{T}^{n}\right]+\frac{\alpha}{2} \sqrt{\operatorname{Var}_{\mathcal{F}_{0}}^{\mathbb{P}^{a} \times \mathbb{P}^{f}}\left(g_{T}^{n}\right)}\right)=\pi_{0}^{a}\left(g_{T}^{n} \mid \mathcal{F}_{0}\right),
\end{aligned}
$$

where $\pi_{0}^{a}$ is the standard deviation principle.

\section{APPENDIX C}

\section{C.1. Three-step premium: Example}

Given the payoff (5.2), the fund, and mortality dynamics, we can now compute the different parts of the three-step premium. For the hedgeable part $H_{T}^{(1)}$, we obtain

$$
\pi_{0}^{f}\left(H_{T}^{(1)} \mid \mathcal{F}_{0}\right)=e^{-r T} \mathbb{E}_{\mathcal{F}_{0}}^{\mathbb{P}^{a} \times \mathbb{Q}^{f}}\left[H_{T}^{n}\right]=\pi_{0}^{f}\left(h\left(S_{T}\right) \mid \mathcal{F}_{0}\right) \cdot \mathbb{E}_{\mathcal{F}_{0}}^{\mathbb{P}^{a} \times \mathbb{P}^{f}}\left[e^{-\int_{0}^{T} \lambda_{s} \mathrm{~d} s}\right] .
$$

Due to the independence of financial and actuarial risks, we find that:

$$
\mathbb{E}_{\mathcal{F}_{0}}^{\mathbb{P}^{a} \times \mathbb{P}^{f}}\left[H_{T}^{(2)}\right]=\mathbb{E}_{\mathcal{F}_{0}}^{\mathbb{P}^{a} \times \mathbb{P}^{f}}\left[H_{T}^{(3)}\right]=\mathbb{E}_{\mathcal{F}_{0}}^{\mathbb{P}^{a} \times \mathbb{Q}^{f}}\left[H_{T}^{(2)}\right]=\mathbb{E}_{\mathcal{F}_{0}}^{\mathbb{P}^{a} \times \mathbb{Q}^{f}}\left[H_{T}^{(3)}\right]=0
$$

For the diversifiable part $H_{T}^{(2)}$, we use the standard deviation principle (2.5):

$$
\begin{aligned}
& \pi_{0}^{a}\left(H_{T}^{(2)}\right)=e^{-r T} \frac{\alpha}{2} \sqrt{\mathbb{E}_{\mathcal{F}_{0}}^{\mathbb{P} a} \times \mathbb{P}^{f}\left[h\left(S_{T}\right)^{2}\right]} \cdot \sqrt{\operatorname{Var}_{\mathcal{F}_{0}}^{\mathbb{P}^{a} \times \mathbb{P}^{f}}\left(\frac{1}{n} \sum_{j=1}^{n}\left(g\left(\tau_{j}\right)-\mathbb{E}_{\mathcal{F}_{0} \vee \mathbb{G}_{T}}^{\mathbb{P} \mathcal{P}^{f}}\left[g\left(\tau_{j}\right)\right]\right)\right)} \\
& =e^{-r T} \frac{\alpha}{2} \sqrt{\mathbb{E}_{\mathcal{F}_{0}}^{\mathbb{P} \times \mathbb{P}^{f}}\left[h\left(S_{T}\right)^{2}\right]} \sqrt{\operatorname{Var}_{\mathcal{F}_{0}}^{\mathbb{P}^{a} \times \mathbb{P}^{f}}\left(\frac{1}{n} \sum_{j=1}^{n}\left(g\left(\tau_{j}\right)-\mathbb{E}_{\mathcal{F}_{0} \vee \mathcal{G}_{T}}^{\mathbb{P}^{a}}\left[g\left(\tau_{j}\right)\right]\right)\right)} \\
& =e^{-r T} \frac{\alpha}{2} \sqrt{\mathbb{E}_{\mathcal{F}_{0}}^{\mathbb{P} a} \times \mathbb{P}^{f}\left[h\left(S_{T}\right)^{2}\right]} \cdot \sqrt{\frac{1}{n^{2}} \operatorname{Var}_{\mathcal{F}_{0}}^{\mathbb{P}^{a} \times \mathbb{P}^{f}}\left(N_{T}-n \cdot e^{-\int_{0}^{T} \lambda_{s} \mathrm{~d} s}\right)} \\
& =e^{-r T} \frac{\alpha}{2} \sqrt{\mathbb{E}_{\mathcal{F}_{0}}^{\mathbb{P} \times \mathbb{P}^{f}}\left[h\left(S_{T}\right)^{2}\right]} \cdot \sqrt{\frac{1}{n^{2}} \mathbb{E}_{\mathcal{F}_{0}}^{\mathbb{P} a} \times \mathbb{P}^{f}\left[\operatorname{Var}_{\mathcal{F}_{0} \vee \mathcal{G}_{T}}^{\mathbb{P a} \times \mathbb{P}^{f}}\left(N_{T}-n \cdot e^{-\int_{0}^{T} \lambda_{s} \mathrm{~d} s}\right)\right]} \\
& =e^{-r T} \frac{\alpha}{2} \sqrt{\mathbb{E}_{\mathcal{F}_{0}}^{\mathbb{P}^{a} \times \mathbb{P}^{f}}\left[h\left(S_{T}\right)^{2}\right]} \cdot \sqrt{\frac{1}{n} \mathbb{E}_{\mathcal{F}_{0}}^{\mathbb{P}^{a} \times \mathbb{P}^{f}}\left[e^{-\int_{0}^{T} \lambda_{s} \mathrm{~d} s}\left(1-e^{-\int_{0}^{T} \lambda_{s} \mathrm{~d} s}\right)\right]} .
\end{aligned}
$$


Note that (C.2) approaches 0 for $n \rightarrow \infty$ (diversifiable risk). ${ }^{6}$ For the unhedgeable and nondiversifiable part $H_{T}^{(3)}$, we define the normally distributed risk factors $X_{T}^{(1)}:=-\int_{0}^{T} \lambda_{S} \mathrm{~d} s$ and $X_{T}^{(2)}:=\ln \left(S_{T} / S_{0}\right)$ representing systematic mortality and financial risk, respectively. We can express the part $H_{T}^{(3)}$ of decomposition (3.1) as

$H_{T}^{(3)}=\frac{1}{n} \sum_{j=1}^{n}\left(\mathbb{E}_{\mathcal{F}_{0} \vee \mathcal{G}_{T}}^{\mathbb{P}^{a} \times \mathbb{Q}^{f}}\left[g\left(\tau_{j}\right)\right]-\mathbb{E}_{\mathcal{F}_{0}}^{\mathbb{P}^{a} \times \mathbb{Q}^{f}}\left[g\left(\tau_{j}\right)\right]\right) \cdot h\left(S_{T}\right)=\frac{1}{n} \sum_{j=1}^{n}\left(e^{X_{T}^{(1)}}-{ }_{T} p_{x}^{j}\right) \cdot h\left(P \cdot e^{X_{T}^{(2)}}\right)$.

Use (4.2) to apply the Esscher transform to compute

$$
\begin{aligned}
& \pi_{0}^{n}\left(H_{T}^{(3)} \mid \mathcal{F}_{0}\right)=e^{-r T} \mathbb{E}_{\mathcal{F}_{0}}^{\mathbb{P}^{a} \times \mathbb{P}^{f}}\left[\frac{1}{n} \sum_{j=1}^{n}\left(e^{X_{T}^{(1)}-\theta^{(1)} \Sigma_{1,1}-{ }_{T} p_{x}^{j}}\right) \cdot h\left(P \cdot e^{X_{T}^{(2)}-\theta^{(2)} \Sigma_{2,2}}\right)\right] \\
& =\mathbb{E}_{\mathcal{F}_{0}}^{\mathbb{P}^{a} \times \mathbb{P}^{f}}\left[\left(e^{X_{T}^{(1)}-\theta^{(1)} \Sigma_{1,1}}-T_{x}^{1}\right)\right] \cdot e^{-r T} \mathbb{E}_{\mathcal{F}_{0}}^{\mathbb{P}^{a} \times \mathbb{P}^{f}}\left[h\left(P \cdot e^{X_{T}^{(2)}-\theta^{(2)} \Sigma_{2,2}}\right)\right] .
\end{aligned}
$$

